\title{
1 Integration of physiologically relevant photosynthetic energy flows into whole 2 genome models of light-driven metabolism
}

3

4 Authors: Jared T. Broddrick ${ }^{1,2}$, Maxwell A. Ware ${ }^{3}$, Denis Jallet ${ }^{3}$, Bernhard O. Palsson ${ }^{2}$ and Graham Peers ${ }^{3}$

5 1. Division of Biological Sciences, University of California, San Diego, La Jolla, CA

6 2. Department of Bioengineering, University of California, San Diego, La Jolla, CA

7 3. Department of Biology, Colorado State University, Fort Collins, CO

9 Abstract

10 Characterizing photosynthetic productivity is necessary to understand the ecological contributions and biotechnology potential of plants, algae, and cyanobacteria. Light capture efficiency and photophysiology have long been characterized by measurements of chlorophyll fluorescence dynamics. However, these investigations typically do not consider the metabolic network downstream of light harvesting. In contrast, genome-scale metabolic models capture species-specific metabolic capabilities but have yet to incorporate the rapid regulation of the light harvesting apparatus. Here we combine chlorophyll fluorescence parameters defining photosynthetic and non-photosynthetic yield of absorbed light energy with a metabolic model of the pennate diatom Phaeodactylum tricornutum. This integration increases the model predictive accuracy regarding growth rate, intracellular oxygen production and consumption, and metabolic pathway usage. Additionally, our simulations recapitulate the link between mitochondrial dissipation of photosynthetically-derived electrons and the redox state of the photosynthetic electron transport chain. We use this framework to assess engineering strategies for rerouting cellular resources toward bioproducts. Overall, we present a methodology for incorporating a common, informative data type into computational models of light-driven metabolism for characterization, monitoring and engineering of photosynthetic organisms.

Keywords: chlorophyll fluorescence, genome-scale modelling, diatom, metabolism, electron transport, 


\section{Introduction}

There is great interest in characterizing light-driven metabolism due to the ecological importance and engineering potential of phototrophic microorganisms and plants. Oxygenic photosynthesis utilizes light energy to generate an oxidized protein complex capable of extracting electrons from water at

32 Photosystem II, while concurrently re-energizing the extracted electron to reduce $\mathrm{NADP}^{+}$at Photosystem throughout the cell.

Light absorption by a photosynthetic cell is not constant. Light fluxes can vary across the day and due to local ecological or climatological features. It is common for photosynthetic microorganisms to absorb more photons than what can be utilized by metabolism during these natural fluctuations in sunlight. If this energy is not dissipated, it results in over reduction of the photosynthetic electron transport chain (ETC). Reactive oxygen species are then formed, causing oxidative damage to proteins, lipids, and nucleic acids (Niyogi, 2000; Dietz et al, 2016). When the damage resulting from excess light capture results in a decrease in photosynthetic efficiency it is termed photoinhibition. The photosystem II (PSII) D1 subunit is the primary photoinhibition target in the photosynthetic ETC (Edelman \& Mattoo, 2008). A complex repair cycle characterized by removal, degradation, and de novo synthesis is constitutively active to counter this damage and it is energetically expensive (Nixon et al, 2005). To prevent photoinhibition, excess energy can be dissipated upstream of the photosynthetic ETC complexes via a variety of mechanisms

47 encompassing nonphotochemical quenching (NPQ), which harmlessly converts excitation energy to heat

48 (Nicol et al, 2019). While this protects the photosynthetic system from oxidative stress, it also reduces the overall efficiency of light-biomass conversion.

Here we coin the term Excess Electron Transport (EET) as an additional important physiological

51 feature at the intersection of photophysiology and bioengineering. This comprises several components 
52 that act either as shunts within the ETC (Jallet et al, 2016b; Ware et al, 2020) or downstream of the

53 photosynthetic machinery within the broader metabolic network. It relieves over reduction of the

54 photosynthetic ETC by dispelling electrons generated by excess light (Jallet et al, 2016b). Usually these

55 reactions are considered metabolically "futile" as the electrons are deposited on elemental oxygen to

56 generate water, for instance. However, they are important in relieving photosynthetic ETC over-reduction.

57 There is interest in the bioengineering field to redirect these electrons away from metabolic futility

58 towards bioproducts of interest, while maintaining the beneficial effects on ETC redox balance (Levering

59 et al, 2015; Lassen et al, 2014). Harnessing excess reductant can convert endogenous carbon sinks, such

60 as carbohydrates, into more energy dense products such as lipids. Indeed, recently it was shown

61 engineered reductant sinks can actually increase carbon fixation and overall photosynthetic efficiency

62 (Santos-Merino et al, 2021). Thus, downregulating evolutionarily beneficial processes for photosynthetic

63 individuals in favor of mass culture productivities offers promising avenues for increasing bioproduct and

64 biofuel efficiency. Quantitative characterization of the push-pull of light capture upstream and dissipation

65 in the metabolic network downstream of the photosynthetic ETC would enable design and optimization

66 of these engineered reductant sinks.

67 Properly accounting for EET facilitates this bioprocess optimization and provides insight into

68 photoprotection strategies. Previous work in photosynthetic microorganisms (diatoms and green algae)

69 used photophysiology parameters derived from chlorophyll fluorescence measurements to estimate EET

70 (Wagner et al, 2006). In this previous framework, EET was calculated as the difference between the total

71 absorbed photons and the excitation energy required for biomass production and cellular maintenance.

72 Additionally, the fraction of total absorbed light energy lost upstream of the photosynthetic ETC was

73 estimated using chlorophyll fluorescence data, which have long been employed to assess phototrophic

74 physiology (Krause \& Weis, 1991). 
Chlorophyll fluorescence primarily quantifies the fate of absorbed light energy directed to PSIl; however, there is evidence of contributions from photosystem I (PSI) as well (Giovagnetti et al, 2015; Pfündel et al, 2013). This excitation energy has three primary fates: it can perform photochemistry at PSII; it can be dissipated as heat through NPQ processes; or it can be dissipated by other, less well characterized non-radiative and fluorescence processes (NO). All of these can be quantified through the use of pulse amplitude modulation (PAM) chlorophyll fluorimetry (Kramer et al, 2004). When these values are normalized to the total excitation energy routed to PSII, they are annotated as the quantum yields Y(II), $Y(N P Q)$ and $Y(N O)$, respectively, the sum of which is always one. These techniques have unveiled the diverse photoprotective strategies employed by photosynthetic microorganisms to include extensive NPQ in the diatom Phaeodactylum tricornutum (Lavaud et al, 2002). However, these important aspects of photosynthesis have not been integrated in to models of total cellular metabolism. employed to characterize and engineer a wide range of biological systems (O'Brien et al, 2013). Constraint-based modeling relies on a reconstruction of the metabolic content of the organism of interest. The resulting computational framework, known as a genome scale model (GEM), can then be used to compute a variety of cellular phenotypes. There have been several, recent advances in the metabolic modeling of photosynthetic organisms to include cyanobacteria (Broddrick et al, 2016, 2019b), green algae (Zuñiga et al, 2017; Chang et al, 2011), and diatoms (Levering et al, 2016). Recent modeling in the diatom Phaeodactlyum tricornutum quantified growth rates, excitation energy partitioning between the photosystems and cross-compartment energetic coupling of the chloroplast and mitochondrion (Broddrick et al, 2019a). However, that study used simplified assumptions regarding light harvesting, 96 possibly affecting the accuracy of absolute fluxes predicted by the model. The metabolic network that 97 underpins GEMs is assembled from the reactant and product stoichiometry of biochemical reactions; thus, 

enable the explicit integration of chlorophyll fluorescence data and EET as a constraint on photosynthetic metabolic processes towards an increased understanding of photoacclimation, photoprotection and bioengineering of phototrophic metabolism.

\section{Results}

\section{Cell physiology of P. tricornutum at low and high light}

P. tricornutum was acclimated and cultured at a high light irradiance of $600 \mu \mathrm{mol}$ photons $\mathrm{m}^{-2} \mathrm{~s}^{-1}$ $(\mathrm{HL}, \mathrm{n}=4)$ and a low light irradiance of $60 \mu \mathrm{mol}$ photons $\mathrm{m}^{-2} \mathrm{~s}^{-1}(\mathrm{LL}, \mathrm{n}=3)$. The range of growth rates for $P$. tricornutum was 0.026-0.029 $(n=3)$ and 0.052-0.053 $(n=4) h r^{-1}$ at $L L$ and $H L$, respectively. Cell volumes for cultures grown at both light levels differed by approximately $10 \%$ (202 \pm 43 versus $184 \pm 47 \mu \mathrm{m}^{3}$ at $\mathrm{HL}$ ( $n=94)$ and LL ( $n=46)$, respectively). Dry cell weight was also similar between the cultures (Table 1$)$.

\begin{tabular}{|c|c|c|c|c|c|c|c|}
\hline & $\begin{array}{c}\text { Growth rate } \\
\left(\mathrm{h}^{-1}\right)\end{array}$ & $\begin{array}{l}\text { Cell volume } \\
\qquad\left(\mu \mathrm{m}^{-3}\right)\end{array}$ & $\begin{array}{l}\text { Cell weight } \\
\text { (pgDW/cell) }\end{array}$ & $\begin{array}{c}\text { Chla } \\
\text { (pg/cell) }\end{array}$ & $\begin{array}{c}\text { Chlc } \\
\text { (pg/cell) }\end{array}$ & Chla/Chlc & $\begin{array}{l}\text { Total chl } \\
\text { (LL:HL) }\end{array}$ \\
\hline Low Light & $0.026-0.029$ & $184 \pm 47$ & $19.1 \pm 2$ & $0.384 \pm 0.006$ & $0.073 \pm 0.001$ & 6.0 & 2.8 \\
\hline High Light & $0.052-0.053$ & $202 \pm 43$ & $20.4 \pm 1.4$ & $0.142 \pm 0.014$ & $0.024 \pm 0.003$ & 5.2 & \\
\hline
\end{tabular}

Table 1. Physiology parameters of $P$. tricornutum acclimated to low and high light.

There were differences in the chlorophyll content of cells adapted to different light regimes, as is

114 (chlc)) at LL was 2.8-fold higher than at $\mathrm{HL}$ (Table 1) and resulted in a 3-fold increase in the cell normalized 115 absorption coefficient ( $a^{*}$ cell, Fig. 1A, B); suggesting light capture efficiency remained constant at both 116 acclimated light conditions. The chla to chlc ratio varied slightly from 6.0 at LL to 5.2 at HL. Overall, the 117 chlorophyll content per cell was consistent with previous observations of photoacclimation in $P$. tricornutum (Nymark et al, 2009; Broddrick et al, 2019a). 


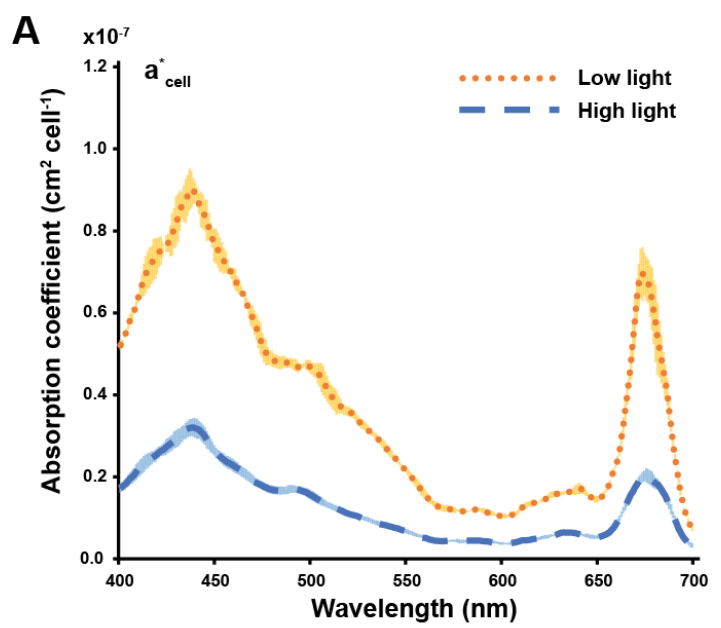

C
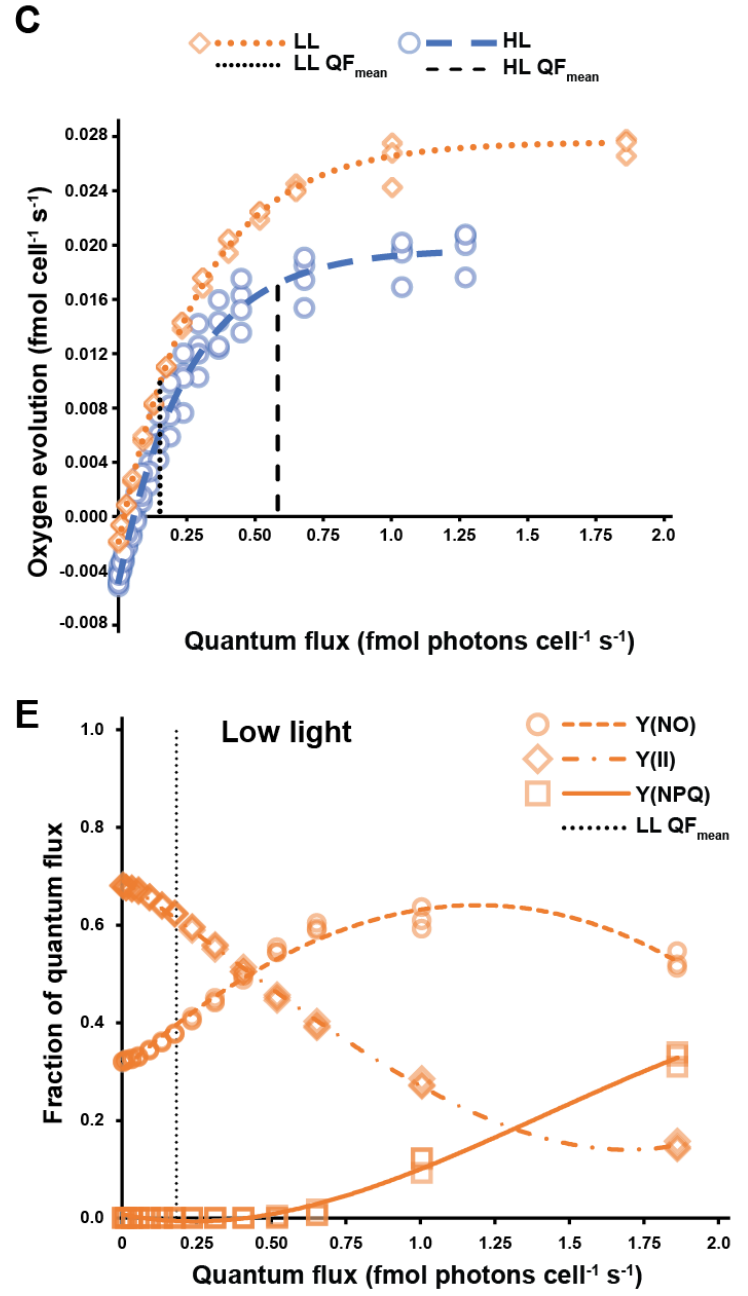

B

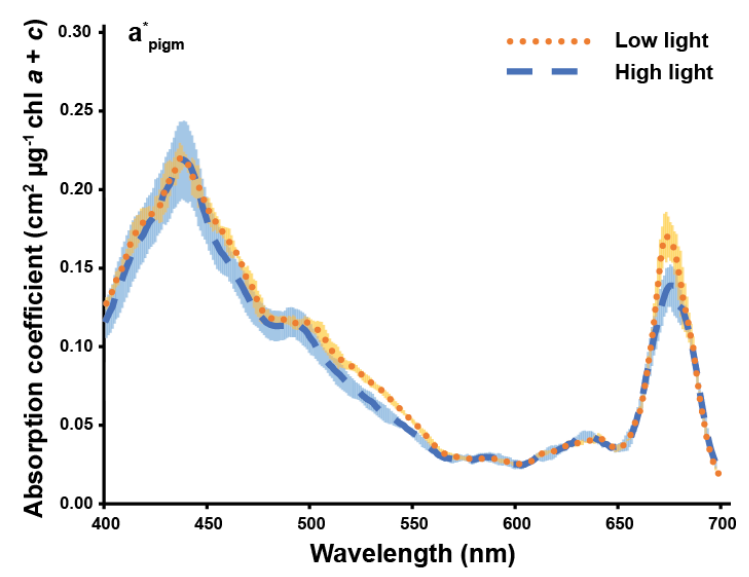

D

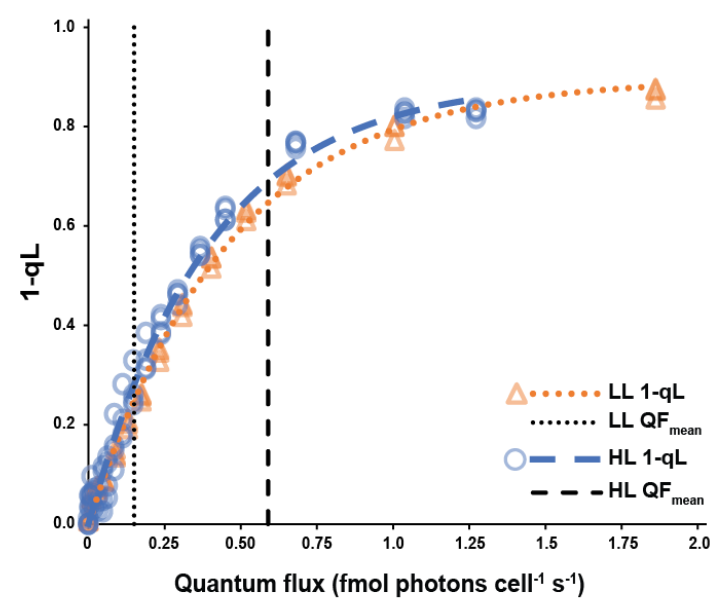

$\mathbf{F}$

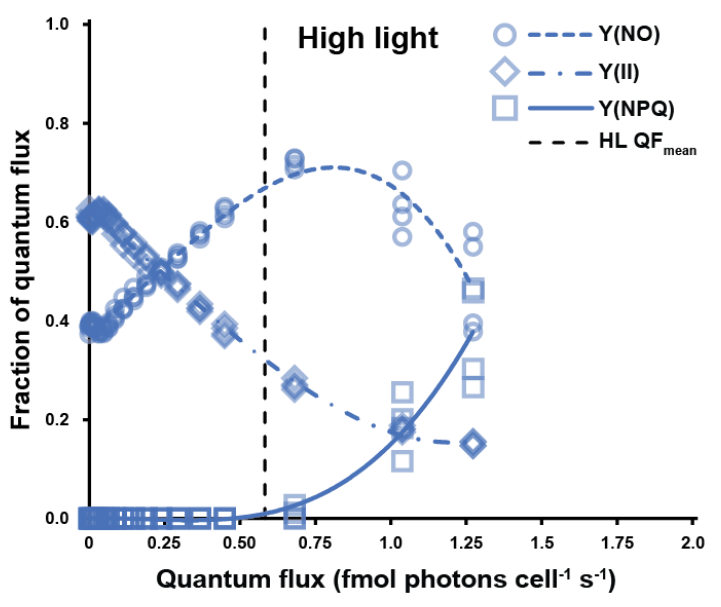

Figure 1. Photophysiology of $\boldsymbol{P}$. tricornutum acclimated to low and high light. (A) Cell-specific absorption coefficient (B) Pigmentspecific absorption coefficient. The pigment mass includes chlorophyll $a$ and chlorophyll $c$. Shaded areas represent one standard deviation from the mean (HL: $n=4, L L: n=3)$. (C) Cell-specific $P_{0}$ versus QF curve. (D) Fraction of closed reaction centers (1-qL) versus QF curve. (E) Chlorophyll fluorescence parameters vs. quantum flux for cells acclimated to low light. (D) Chlorophyll fluorescence parameters vs. quantum flux for cells acclimated to high light. Vertical dashed lines represent the mean quantum flux received by the cultures at the experimental irradiance. Abbreviations and definitions: LL: low light, HL: high light, QF: quantum flux, Y(II): quantum efficiency of photosystem II, NPQ: non-photochemical quenching, $Y(N O)$ : unregulated, non-radiative dissipation of excitation energy. Data based on $n=3$ biological replicates for $L L$ and $n=4$ biological replicates for $H L$. 

constrained with the photon uptake rate (quantum flux, QF) and the oxygen evolution rate in a manner similar to recent modeling efforts in cyanobacteria and diatoms (Broddrick et al, 2019a, 2019b). capture rate across the full path length. As PAM measurements require high cell densities to generate sufficient fluorescence signal, we used a similar approach to account for cell shading in the PAM sample cuvette (Broddrick et al, 2019b). This calculated $Q F_{\text {mean }}$ was used as the independent variable for Po vs. QF curves as well as plots of chlorophyll fluorescence parameters vs. QF.

143 (Table 2, $\mathrm{Po}_{\text {mean }}$ ), quantifying the impact of self-shading from the increased pigment content at LL on overall productivity. The $P_{0}$ vs. QF curve initial slopes were $9.5 \times 10^{-2}$ and $9.8 \times 10^{-2} \mathrm{~mol} \mathrm{O}_{2} \mathrm{~mol} \mathrm{photon}^{-1}$ for photon ${ }^{-1} \mathrm{~m}^{2} \mathrm{mgChl}^{-1}$ for both HL and LL (Fig. S1).

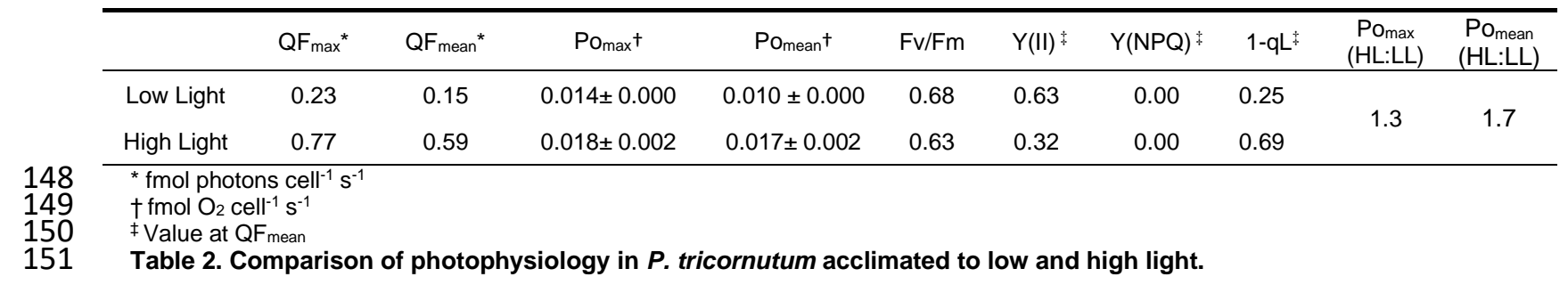


Overall, trends in the chlorophyll fluorescence parameters plotted against quantum flux (PAM vs.

153 QF) were similar between the two light regimes (Fig. 1D-F). The effective quantum yield of PSII, [Y(II)],

154 decreased rapidly with increasing QF for both light regimes. Non-photochemical quenching, contributed

155 almost no excitation energy dissipation at either HL or LL (Table 2 and Fig. 1E, F). As Y(II) accounts for the

156 fraction of QF performing photochemistry and $\mathrm{Y}(\mathrm{NPQ})$ is the fraction of QF lost as heat, the balance, $\mathrm{Y}(\mathrm{NO})$,

157 accounts for the remaining fraction of QF that is dissipated in unregulated, non-radiative processes. $Y$ (NO)

158 accounted for approximately $68 \%$ and $37 \%$ of PSII-directed excitation energy at QF mean for HL and LL,

159 respectively. The fraction of closed reaction centers (1-qL) is a proxy for the redox state of the

160 plastoquinone pool and it was almost identical across the entire QF range for $\mathrm{HL}$ and $\mathrm{LL}$ acclimated

161 samples (Fig. 1D). Additionally, NPQ had similar activation profiles in both LL and HL acclimated cultures.

162 For both $\mathrm{LL}$ and $\mathrm{HL}$ acclimated cultures, $\mathrm{Y}(\mathrm{NPQ})$ activated at a QF of approximately $0.7 \mathrm{fmol}^{\text {photons cell }}{ }^{-1}$

$163 \mathrm{~s}^{-1}, 1-\mathrm{qL}$ values of $0.70-0.75$ and approximately $90 \%$ of their respective maximum photosynthetic rates

164 (Fig. S2).

The cell densities used, and photosynthetic rates observed, at high light suggested carbon

166 limitation in the samples during analysis. Initially, we did not supplement the PAM samples with

167 bicarbonate as we were trying to assess the photophysiology of the experimental culture, which was only

168 sparged with air. However, to assess the possibility of carbon limitation and its influence on light capture

169 efficiencies, we repeated the $P_{O}$ vs. QF and PAM experiments supplementing the samples with $5 \mathrm{mM}$

170 bicarbonate. The resulting differences in $P_{0}$ Vs. QF for $P$. tricornutum suggested a $15 \%$ underestimation of

171 oxygen evolution capacity at $\mathrm{QF}_{\text {mean }}$ for the $\mathrm{HL}$ acclimated condition, but additional bicarbonate

172 supplementation had no impact on the LL condition. (Fig. S3A, B). Additionally, PAM results were

173 comparable between samples with and without added bicarbonate (Fig. S3C), suggesting the carbon

174 limitation was minor enough to not affect quantum efficiency.

175 Simulating photoautotrophic growth of P. tricornutum at low and high light 
We simulated photoautotrophic growth at low and high light by translating the photophysiology

177 results into modeling constraints (Broddrick et al, 2019a, 2019b). The photon uptake rate for the model

178 was derived from $\mathrm{a}^{*}{ }_{\text {cell }}$ coupled with the experimental PAR intensity and the emission spectrum of the

179 fluorescent light used during culturing. This value, equivalent to $Q F$, was used to determine the oxygen

180 evolution rate of the culture (using the curves in Fig. 2), which constrained the oxygen exchange reaction

181 for the simulations.

Next, we incorporated the chlorophyll fluorescence data with our modeling construct. GEMs are built using biochemical reaction stoichiometry. Chlorophyll fluorescence parameters are normalized as

fractional values of excitation energy routed to photosystem II, which can be formulated in a similar manner to stoichiometry in canonical biochemical reactions. Thus, we added a pseudo-reaction to the model that imposes this fractionation between excitation energy lost in the pigment bed $\left(1-F_{v} / F_{m}\right)$, 187 photochemical yield $[\mathrm{Y}(\mathrm{II})]$, regulated non-photochemical quenching [Y(NPQ)], and unregulated nonphotochemical quenching $[\mathrm{Y}(\mathrm{NO})]$ (Fig. S4). When the model simulates photoautotrophic growth, it predicts the excitation energy split between the photosystems to satisfy the reductant and ATP needs for biomass production. The chlorophyll fluorescence parameters apply a constraint on the excitation energy split as only the Y(II) fraction can perform photochemistry at PSII.

A final constraint added to the model accounted for photodamage of the PSII D1 subunit. We determined the D1 damage rate at the experimental irradiance for both $\mathrm{LL}$ and $\mathrm{HL}$ acclimated cells by comparing the maximum quantum yield of PSII $\left(\mathrm{F}_{\mathrm{v}} / \mathrm{F}_{\mathrm{m}}\right)$ with and without lincomycin, a plastid protein synthesis inhibitor (Fig. S5A, B). We determined the D1 damage first-order rate constant to be between $5 \times 10^{-4}$ and $7 \times 10^{-4}(n=3)$ and $2.22 \times 10^{-2}$ and $2.52 \times 10^{-2}(n=3)$ for $L L$ and $H L$ acclimated samples, respectively.

197 Through Western blot analysis we determined the D1 protein to be approximately $1.1 \%$ of total protein 
values were added as a maintenance energy reaction to the model requiring $360 \mathrm{mmol}$ ATP and $720 \mathrm{mmol}$

201 GTP to be consumed at the plastid ribosome to polymerize $1 \mathrm{mmol}$ of D1 protein. A summary of these values can be found in Table S1.

With the suite of photophysiology constraints incorporated into the GEM, we simulated photoautotrophic growth for both light conditions. To account for experimental variability on the model predictions, we simulated growth using a parameter space that included the mean and plus or minus one standard deviation of the experimentally determined $P_{0}$ vs. QF curves (Fig. 1C), a* cell (Fig 1A) and dry cell weight (Table 1). Simulated growth rates were consistent with experimental values (Fig. 2). The model spiked data (Fig. S3A) resulted in a simulated mean growth rate of $0.052 \pm 0.005$ (Fig. 6).

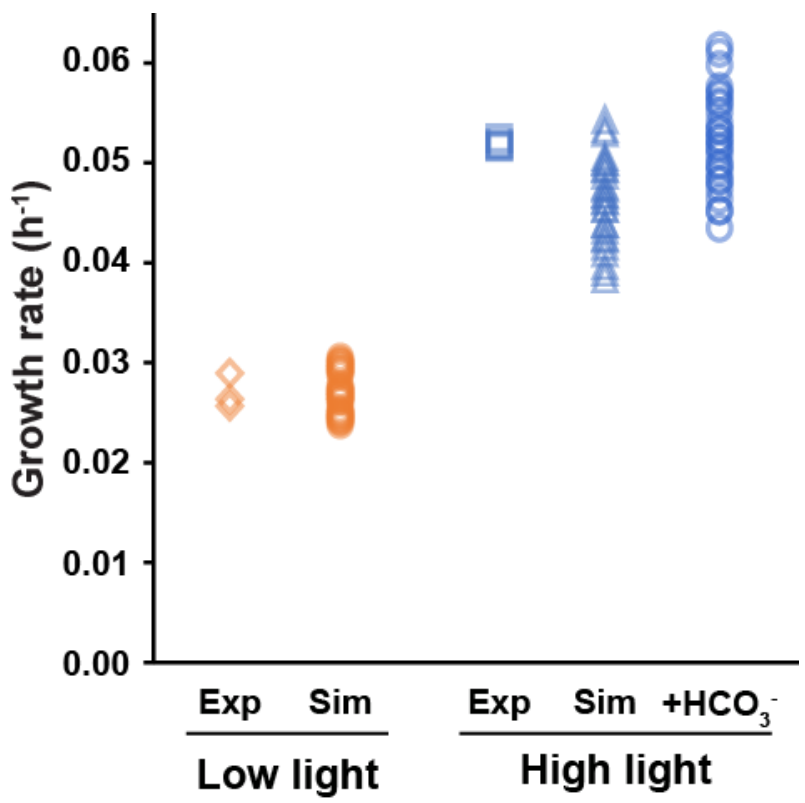
represent the growth rate for independent biological replicates (HL: $n=4, L L: n=3$ ). For the simulation values, the data points represent the simulated growth rate using the mean, +1 standard deviation and -1 standard deviation of the $\mathrm{O}_{2}$ vs QF curves, $\mathrm{a}_{\text {cell }}^{*}$ and cell dry weight ( $\mathrm{n}=27$ parameter combinations). Abbreviations. Exp: experimental, Sim: simulated, $+\mathrm{HCO}_{3}:$ simulated, bicarbonate spiked. 
We assessed the relative contribution of various constraints on the accuracy of model predictions, specifically photon uptake, oxygen evolution, photosystem II repair requirements and photochemical yield as constraints on growth rate (Table 3). For the LL simulations, the model was parameterized with the mean values for $\mathrm{Po}_{\mathrm{V}}$ Vs. QF, a* ${ }_{\text {cell }}$ and dry cell weight. For the $\mathrm{HL}$ simulations, we used the mean values for all but $P_{0}$ vs. QF, where we used the bicarbonate spike-adjusted data, as it most accurately represented the photophysiology of the experimental cultures (Fig. 2).

\begin{tabular}{lc|c} 
& \multicolumn{2}{c}{$\boldsymbol{P}$. tricornutum } \\
\cline { 2 - 3 } & $\mathrm{HL}$ & $\mathrm{LL}$ \\
\hline Experimental & $0.052-0.053$ & $0.026-0.029$ \\
$h v$ & 0.136 & 0.038 \\
Po & 0.052 & 0.027 \\
$h v$, Po, D1 & 0.052 & 0.027 \\
$h v, \mathrm{Y}(\mathrm{II})$ & 0.062 & 0.030 \\
$h v, \mathrm{Po}, \mathrm{D} 1, \mathrm{Y}(\mathrm{II})$ & 0.052 & 0.027 \\
$h v, \mathrm{Po}, \mathrm{DM} 2 \%$ & 0.052 & 0.027 \\
\hline
\end{tabular}

Table 3: Hierarchy of constraints for $\boldsymbol{P}$. tricornutum simulations. Abbreviations: $h v-$ photon uptake, $\mathrm{P}_{\mathrm{O}}-$ oxygen evolution rate, D1 - PSII D1 protein repair, Y(II) - PSII quantum yield, DM20\% - previous modeling assumption of $20 \%$ of absorbed photons lost upstream of the photosystems (Broddrick et al, 2019a). Values are given in units of $\mathrm{h}^{-1}$.

\section{Constraining D1 repair and Y(II) affects metabolic pathway predictions in P. tricornutum}



metabolic pathway usage. Intracellular reaction fluxes provide insight into the metabolic phenotype and form the basis of GEM-based bioengineering strategies. In previous modeling efforts in P. tricornutum

244 acclimated to high light, the predicted rate of intracellular oxygen consumption was higher than that

245 which was observed experimentally (Broddrick et al, 2019a). The previous modeling effort assumed up to

$24620 \%$ of the photon flux could be dissipated upstream of the photosystems. While this assumption did not

247 affect the predicted growth rate (Table 3), we hypothesized the over-estimation of intracellular oxygen

248 consumption could affect metabolic pathway activation and absolute flux values. Additionally, D1 protein

249 repair cost was not included in these previous model simulations. Thus, we simulated photoautotrophic

250 growth in $P$. tricornutum with both the previous $20 \%$ assumption and with our experimentally derived

$251 \mathrm{Y}(\mathrm{II})$ values and D1 damage rates; comparing the model predictions with experimental $\mathrm{O}_{2}$ exchange values

252 derived from membrane inlet mass spectrometry (MIMS).

We defined the model predicted intracellular oxygen consumption as the ratio of the oxygen evolution and the PSII oxygen generation rates. For the LL acclimated condition, both the $Y(I I)$ and $20 \%$ assumption resulted in almost identical predictions of intracellular $\mathrm{O}_{2}$ consumption $[24-33 \%$ and $24-35 \%$ of total PSII $\mathrm{O}_{2}$ generation for the $\mathrm{Y}(\mathrm{II})$ constrained versus the $20 \%$ assumption, respectively; ranges based on Flux Variability Analysis (FVA)]. These values were consistent with previously reported experimentally

258 determined values in $\mathrm{LL}$ acclimated $P$. tricornutum of $35 \pm 5 \%$ (Broddrick et al, 2019a). For $P$. tricornutum

259 acclimated to $\mathrm{HL}$, the predicted intracellular oxygen consumption values were dramatically different 260 between the $20 \%$ assumption and the $Y$ (II) constrained simulation (63-67\% and $40-46 \%$ respectively; 261 ranges based on FVA). Using MIMS, we experimentally determined the fraction of PSII $\mathrm{O}_{2}$ generation 262 consumed by light-independent mechanisms (maintenance), consumed by light-dependent mechanisms 263 (EET), and evolved (net $\left.\mathrm{P}_{\mathrm{O}}\right)$ by cells acclimated to high light $\left(600 \mu \mathrm{mol}\right.$ photons $\left.\mathrm{m}^{-2} \mathrm{~s}^{-1}\right)$. For this HL condition, 264 we determined $42 \pm 5 \%$ of the PSII generated $\mathrm{O}_{2}$ was consumed via intracellular consumption, compared 
to the $Y(I I)$ constrained simulation range of $40-46 \%$. Additionally, the MIMS experiment determined

271 Table 4 summarizes the Y(II) constrained model predicted photosynthetic parameters.

\begin{tabular}{|c|c|c|c|c|c|c|c|c|}
\hline & \multirow[b]{2}{*}{$\mathrm{QF}^{1}$} & \multirow[b]{2}{*}{$\mathrm{ETR}^{2}$} & \multirow[b]{2}{*}{$\begin{array}{l}\text { Quantum } \\
\text { demand }^{3}\end{array}$} & \multicolumn{2}{|c|}{ Fraction of QF } & \multirow{2}{*}{$\begin{array}{c}\begin{array}{c}\text { Charge } \\
\text { Separations }\end{array} \\
\text { PSI/PSII }\end{array}$} & \multirow[b]{2}{*}{$\Phi \mathrm{CO}_{2}{ }^{4}$} & \multirow[b]{2}{*}{$\Phi \mathrm{O}_{2}{ }^{4}$} \\
\hline & & & & PSII & PSI & & & \\
\hline High light & $6.1 \times 10^{-10}$ & $1.4 \times 10^{-10}$ & 16.4 & 0.76 & 0.24 & 0.97 & 0.023 & 0.033 \\
\hline Low light & $1.3 \times 10^{-10}$ & $0.5 \times 10^{-10}$ & 10.5 & 0.60 & 0.40 & 1.05 & 0.044 & 0.066 \\
\hline \multicolumn{9}{|c|}{${ }^{1} \mu \mathrm{mol}$ photon celll $\mathrm{s}^{-1}$} \\
\hline \multicolumn{9}{|c|}{${ }^{2} \mu \mathrm{mol}$ electron cell ${ }^{-1} \mathrm{~s}^{-1}$} \\
\hline \multicolumn{9}{|c|}{${ }^{3} \mathrm{QF} \times(0.25 \times \mathrm{ETR})^{-1}$; mol photon $\times \mathrm{mol}^{-1} \mathrm{O}_{2}$} \\
\hline${ }^{4} \mu \mathrm{mol} \times \mu \mathrm{mo}$ & Shoton & & & & & & & \\
\hline
\end{tabular}

Table 4. Predicted excitation energy flow in $\boldsymbol{P}$. tricornutum acclimated to low and high light. Values are for simulations constrained to account for $100 \%$ of absorbed quanta. $\Phi \mathrm{CO}_{2}$ : quantum yield of net carbon fixation, $\Phi_{2}$ : quantum yield of net oxygen evolution. Abbreviations: QF: quantum flux, ETR: electron transport rate, PSII: photosystem II, PSI: photosystem I, Y(NO): unregulated excitation energy dissipation, NPQ: non-photochemical quenching.

Next, we investigated how incorporating chlorophyll fluorescence data affected predictions of cross-compartment coupling. Previous modeling in $P$. tricornutum hypothesized excess reductant was shunted from the chloroplast to the mitochondrion (Broddrick et al, 2019a), consistent with experimental evidence of energetic coupling of these compartments (Bailleul et al, 2015; Murik et al, 2019). This previous work suggested photorespiration, branch-chain amino acids, and an ornithine-mediated chloroplast-mitochondrion shunt were the dominant mechanisms for cross-compartment coupling (Broddrick et al, 2019a). However, the Y(II) constrained model, validated with the $\mathrm{O}_{2}$ values from the MIMS experiment, suggested $P$. tricornutum acclimated to high light has a substantially reduced effective quantum yield at the experimental irradiance [Y(II), Table 2]. Thus, excess excitation energy not performing photochemistry (e.g. NPQ) likely reduces the need for cross-compartment coupling. 

excitation energy assumption (hereafter $20 \%$ assumption) and the combined $Y(I I)$ and D1 repair

NPQ as QF increased (Fig 2).
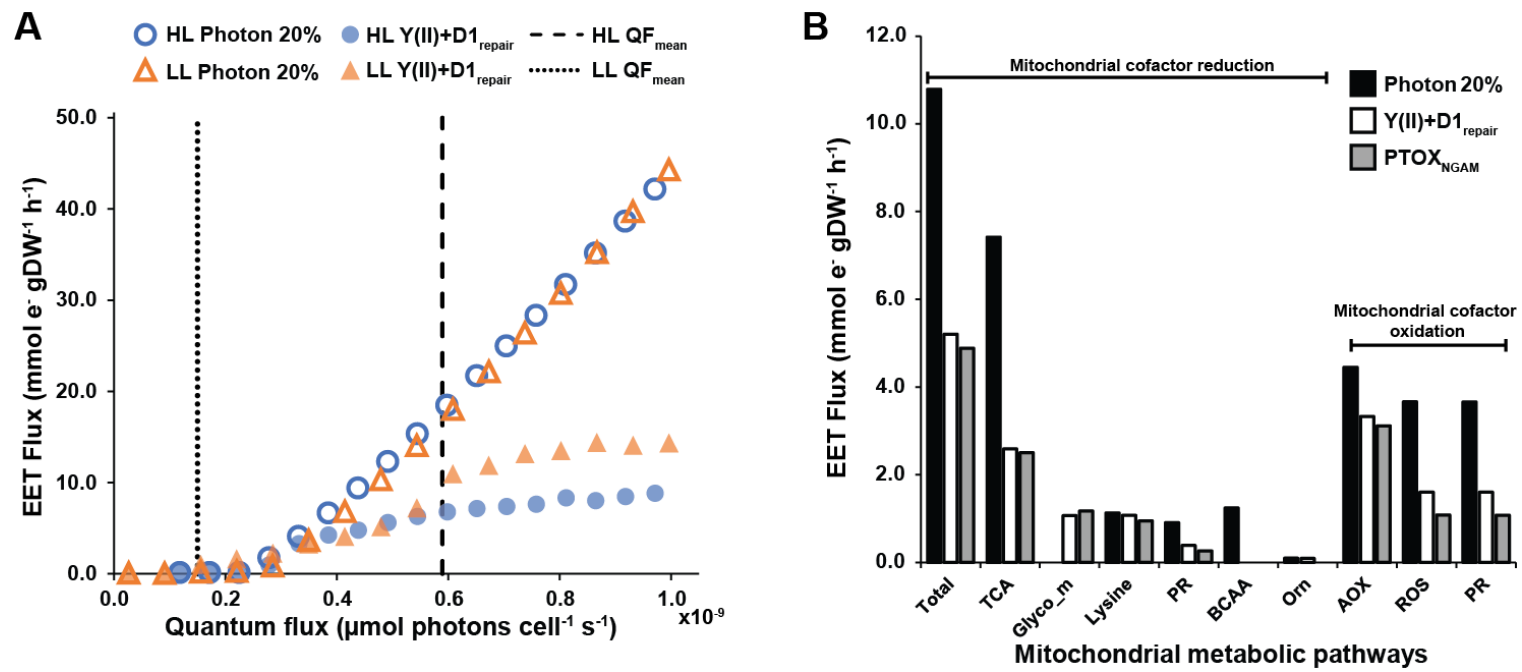

Figure 3. Chlorophyll fluorescence and D1 damage constraints affects model predictions for cross-compartment metabolic coupling. (A) Predicted EET as a function of quantum flux for cells acclimated to LL (triangles) or HL (circles). Open markers: simulations where a fixed $20 \%$ of captured photons are lost upstream of the photosystems; filled markers: simulations with Y(II) and D1 repair constraints. Vertical dashed lines represent the mean quantum flux received by the cultures at the experimental irradiance. (B) Total metabolic flux shunted to the mitochondrion via different metabolic pathways for $P$. tricornutum acclimated to high light. Black bars: Simulations where a fixed $20 \%$ of captured photons are lost upstream of the photosystems; white bars: simulations with Y(II) and D1 repair constraints; gray bars: simulations with $Y(I I)$ and D1 repair constraints and NGAM routed to PTOX. Abbreviations: AOX: alternative oxidase; BCAA: branched-chain amino acid; Glyco_m: mitochondrial glycolysis; Orn: ornithine shunt; PR: photorespiration (reducing: glycine cleavage system; oxidizing: glyoxylate transaminases); PTOX: plastid terminal oxidase; ROS: reactive oxygen species detoxification; TCA: mitochondrial tricarboxylic acid cycle. 
311 for cells acclimated to HL. The $20 \%$ assumption predicted $107 \%$ more photosynthetically derived electrons

312 were shuttled to the mitochondria compared to the $Y(I I)$ constrained simulation. This difference in cross-

313 compartment energetic coupling resulted in similar cross-compartment shuttles as previously reported

314 (Broddrick et al, 2019a); however, the absolute flux through these pathways was altered (Fig. 3B). The

$315 Y$ (II) constrained simulations predicted decreased flux through all pathways, apart from mitochondrial

316 glycolysis, compared to the $20 \%$ assumption simulations (Fig 3B). We also explored whether the

317 compartmentalization of light-independent $\mathrm{O}_{2}$ consumption [non-growth associated maintenance

318 (NGAM)] affected EET predictions. When we constrained NGAM to the plastid terminal oxidase (PTOX), a

319 thylakoid membrane-localized EET reaction, there was a slight decrease in absolute flux values predicted

320 in mitochondrial EET pathways commensurate with the reduction in photosynthetically derived electrons

321 leaving the plastid ETC. However, the overall trends were consistent with mitochondrial targeted NGAM

322 simulations. An unexplored, potential cross-compartment shuttle suggested by these simulations was the

323 amino acid lysine. However, lysine catabolic flux in the mitochondrion was similar for all conditions (Fig.

324 3B) suggesting this particular cross-compartment shuttle may not be used as an EET pathway.

327 reduced carbon skeletons (glutamate and alanine) to more oxidized forms (alpha ketoglutarate and 328 pyruvate) during glyoxylate transaminase reactions. These last two categories function to detoxify

329 glycolate produced via photorespiration. A unique feature of photorespiration is that it performs both 330 reduction and oxidation of mitochondrial cofactors. Glycine produced by transaminase reactions during 331 the detoxification of glycolate was predicted to be consumed by the glycine cleavage system producing $332 \mathrm{NADH}$. This reductant then helped fuel the AOX reaction. This linkage between photorespiration, ROS, 333 and AOX is consistent with studies showing AOX to be activated by ROS stress and important in 

caveat as previous modeling efforts in $P$. tricornutum: experimentally determined intracellular fluxes for this organism have not been adequately determined (e.g., ${ }^{13} \mathrm{C}$ metabolic flux analysis) and as such our flux

337 predictions are not yet validated.

338 $\mathrm{gDW}^{-1} \mathrm{~h}^{-1}$ (Table 5).

\section{Model-based exploration of bioengineering potential}

GEMs account for every known biochemical reaction in the organism and can calculate accurate assessments of resource requirements for biomass components and bioproducts (Dinh et al, 2018). We calculated the fraction of linear electron transport (LET) used to biosynthesize each biomass macromolecular fraction (Table 5). The results provided insight into the relative reductant cost of each macromolecular component, which ranged from $177 \mathrm{mmol} \mathrm{e}^{-} \mathrm{gDW}^{-1}$ of carbohydrate to $411 \mathrm{mmol} \mathrm{e}^{-} \mathrm{gDW}^{-}$

${ }^{1}$ of membrane lipids. Using these values and the amount of excess photosynthetically generated reductant in the system, we calculated the theoretical yield of different biomass components if that EET could be rerouted for biomass biosynthesis. The predictions were inversely related to reductant cost with $20.0 \mathrm{mg}$ carbohydrates $\mathrm{gDW}^{-1} \mathrm{~h}^{-1}$ being the highest yield and membrane lipids the lowest at $8.6 \mathrm{mg}$ lipids

\begin{tabular}{|c|c|c|c|c|}
\hline $\begin{array}{l}\text { Biomass } \\
\text { component }\end{array}$ & $\begin{array}{l}\text { Biomass } \\
\text { percent }\end{array}$ & \%LET & $\begin{array}{l}\text { Reductant } \\
\text { cost }^{3}\end{array}$ & $\begin{array}{l}\text { Theoretical } \\
\text { yield }^{4}\end{array}$ \\
\hline Protein & 70.0 & 43.3 & 304 & 11.6 \\
\hline Structural carb & 6.0 & 2.2 & 177 & 20.0 \\
\hline DNA & 0.3 & 0.2 & 254 & 13.9 \\
\hline Membrane lipids & 3.0 & 2.5 & 411 & 8.6 \\
\hline Pigments & 2.2 & 1.7 & 388 & 9.1 \\
\hline Plastid lipids & 3.7 & 2.6 & 347 & 10.2 \\
\hline RNA & 2.7 & 1.4 & 253 & 14.0 \\
\hline Storage $^{1}$ & 10.0 & 5.2 & 258 & 13.7 \\
\hline EET & $\mathrm{N} / \mathrm{A}$ & 29.1 & $\mathrm{~N} / \mathrm{A}$ & $\mathrm{N} / \mathrm{A}$ \\
\hline Other ${ }^{2}$ & $\mathrm{~N} / \mathrm{A}$ & 11.9 & $\mathrm{~N} / \mathrm{A}$ & $\mathrm{N} / \mathrm{A}$ \\
\hline
\end{tabular}


${ }^{4}$ Milligram dry weight component per gram dry weight biomass per hour ( $\mathrm{mg} \mathrm{gDW}^{-1} \mathrm{~h}^{-1}$ ) Table 5. Photosynthetically generated electron requirements for different biomass components for cells acclimated to high light. Abbreviations: LET: linear electron flow; EET: alternative electron transport.

Finally, we explored model-driven engineering strategies to produce high value bioproducts. Bioengineering of photosynthetic microbes has typically targeted fuel or nutraceutical precursors and these targets are normally derived from three different precursors - fatty acids, aromatic amino acids and terpenoids (Brey et al, 2020; Kumar et al, 2020). We evaluated engineering intracellular pathways to increase flux through plastid fatty acid biosynthesis (hexadecanoate), the shikimate pathway (chorismate), and isoprenoid precursors (isopentenyl pyrophosphate). In our simulation, downregulation of EET provided extra reductant for bioproducts; however, carbon and other elements were also diverted from other biomass components. We simulated diverting up to $50 \%$ of cellular biomass to bioproduct synthesis, in increments of $10 \%$, and evaluated changes in the intracellular reaction fluxes that could enable lightdrive production of these compounds (Fig 5).
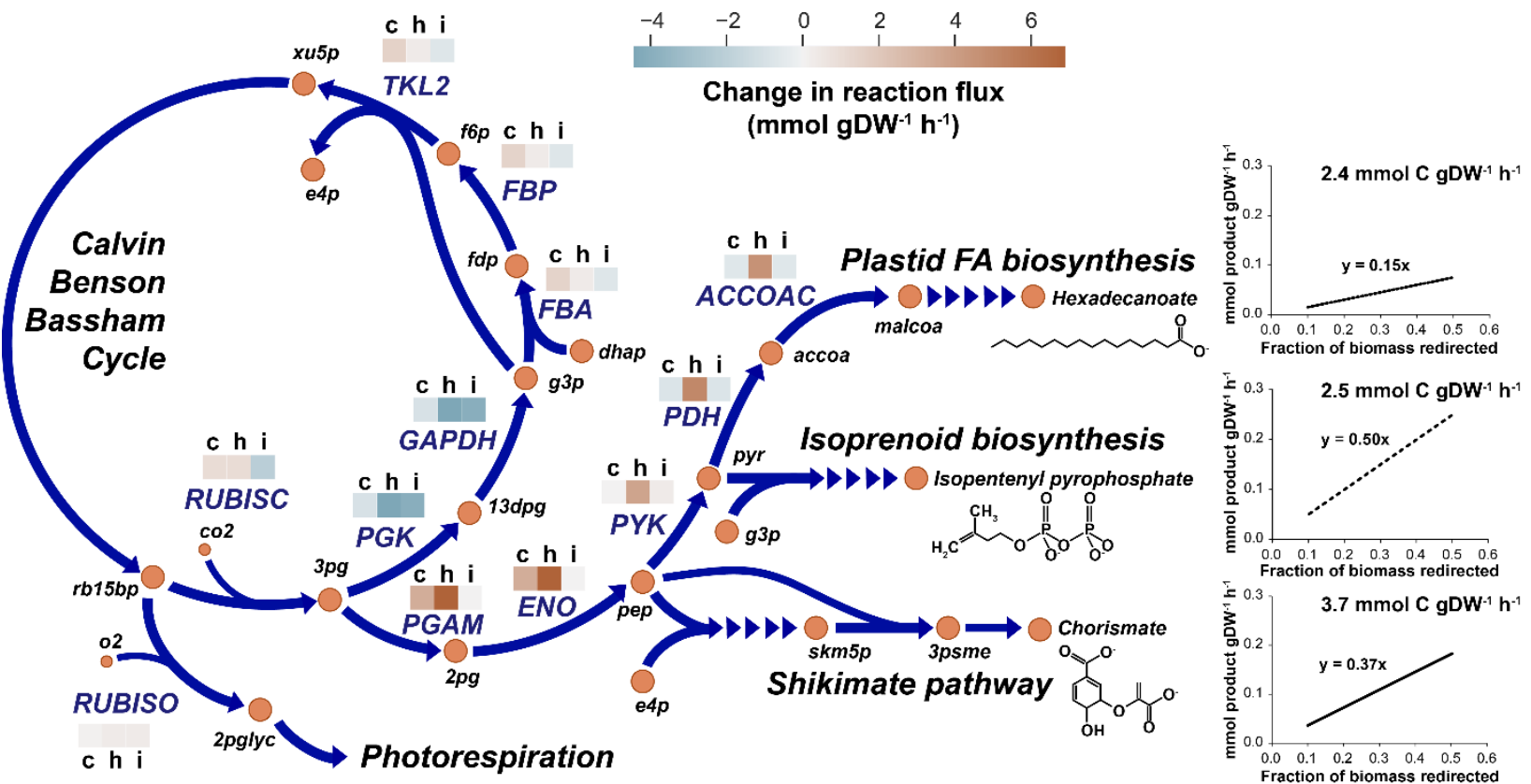

Fig 5. Metabolic engineering potential of $\boldsymbol{P}$. tricornutum acclimated to high light. Changes in metabolic reaction flux towards the bioproducts hexadecanoate, isopentenyl pyrophosphate and chorismate are shown on the flux map. The heatmap above the reactions indicate an increase or decrease in flux towards chorismate (c), hexadecanoate (h), or isopentenyl pyrophosphate (i). Values represent the difference between the baseline simulation fluxes [Y(II) constrained] and bioproduct formation with $30 \%$ of biomass rerouted to the desired product. Graphs indicate bioproduct yield as a function of \%biomass diverted as well as the carbon-normalized yields. Abbreviations are based on the BiGG Models database (King et al, 2016). Abbreviations (Reactions): ACCOAC- acetyl-CoA carboxylase; ENO- enolase; FBA- fructose-1,6-bisphosphate aldolase; FBP- fructose-1,6-bisphosphase; GAPDH-glyceraldehyde-3phosphate dehydrogenase; PDH- pyruvate dehydrogenase; PGAM- phosphoglycerate mutase; PGK- phosphoglycerate kinase; PYK- 
pyruvate kinase; RUBISC- ribulose-1,5-bisphophate carboxylase; RUBISO- ribulose-1,5-bisphophate oxygenase; TKL2transketolase 2. Abbreviations (metabolites): 13dpg- D-glycerate 1,3-diphosphate; 2pg- 2-phospho-D-glycerate; 2pglyc- 2phosphoglycolate; 3pg- 3-phospho-D-glycerate; 3psme- 5-O-(1-carboxyvinyl)-3-phosphoshikimate; accoa- acetyl-CoA; co2- carbon dioxide; dhap- dihydroxyacetone phosphate; e4p- D-erythrose-4-phosphate; f6p- beta-D-fructose 6-bisphosphate; fdp- beta-Dfructose 1,6-bisphosphate; g3p- glyceraldehyde-3-phosphate; malcoa- malonyl-CoA; o2- oxygen; pep- phosphoenolpyruvate; pyrpyruvate; rb15bp- ribulose-1,5-bisphosphate; skm5p- shikimate-5-phosphate; xu5p- D-xyulose 5-phosphate.

The model predicted a linear increase in product yield as a function of increased biomass diverted

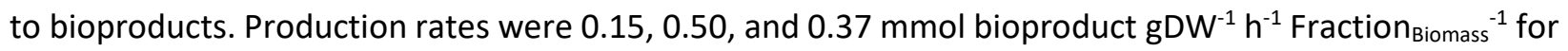
hexadecanoate, isopentenyl pyrophosphate, and chorismate, respectively. When normalized to the number of carbons in each of these end products, the yields were $2.4,2.5$, and $3.7 \mathrm{mmol} \mathrm{C}$ fixed in bioproduct $\mathrm{gDW}^{-1} \mathrm{~h}^{-1}$ Fraction $_{\text {Biomass }}{ }^{-1}$ for hexadecanoate, isopentenyl pyrophosphate, and chorismate, respectively. There were no major differences in predicted EET as a result of bioproduct synthesis. We compared the baseline EET [Y(II) constrained simulations above] to the EET of the production strains with $30 \%$ of biomass diverted to bioproduct synthesis. The baseline EET was $3.13 \mathrm{mmol} \mathrm{e}^{-} \mathrm{DW}^{-1} \mathrm{~h}^{-1}$ compared to $3.08,3.21$, and $3.09 \mathrm{mmol} \mathrm{e}^{-} \mathrm{gDW}^{-1} \mathrm{~h}^{-1}$ hexadecanoate, isopentenyl pyrophosphate, and chorismate, respectively. This result suggested the reductant cost of hexadecanoate and chorismate are higher than the mean biomass reductant cost, while the reductant cost of isopentenyl pyrophosphate is lower.

The flux simulations identified metabolic pathways where rerouting of flux is required for bioproduct synthesis. We compared model-predicted metabolic flux routing at an intermediate biomass diversion value of $30 \%$. All three metabolites, hexadecanoate, isopentenyl pyrophosphate, and chorismate, require plastid glycolytic precursors (acetyl-CoA, pyruvate, and phosphoenolpyruvate, respectively). Additionally, chorismate and isopentenyl pyrophosphate both require Calvin-BensonBassham Cycle (CBBC) intermediates (D-erythrose-4-phosphate and glyceraldehyde-3-phosphate, respectively) for biosynthesis. These requirements were evident in the reaction flux differences between the reference simulation [Y(II) constrained] and the bioproduct simulations (Fig 5). Hexadecanoate biosynthesis required the largest flux rerouting through lower plastid glycolysis as all the carbon required for its biosynthesis is sourced from acetyl-CoA. For chorismate, six of its ten carbons come from lower 
glycolysis, requiring increased flux through the reactions phosphoglycerate mutase and enolase. The remaining four carbons are sourced through the $\mathrm{CBBC}$ resulting in a slight increase in flux through these reactions, to include a predicted increase in carbon fixation at RUBSICO. While initiation of isopentenyl pyrophosphate biosynthesis utilizes the lower glycolytic metabolite pyruvate, the model predicted the source of this metabolite was recycling of carbon from plastid-mitochondrial metabolic coupling, not redirection of flux away from the CBBC. This result shows how GEMs can result in non-intuitive flux routing towards engineering bioproducts.

\section{Discussion}

In this study, we characterized photoautotrophic metabolism in P. tricornutum through integrated chlorophyll fluorescence measurements and genome-scale modeling. Our observations in P. tricornutum were consistent with photophysiology under fluctuating and sinusoidal light (Wagner et al, 2006) and photoacclimation (Nymark et al, 2009). P. tricornutum exhibited efficient photoacclimation with the quanta absorbed per pigment remaining consistent between low and high light (Fig. 1B). This efficiency 415 was also observed when looking at the initial slope of the cell-normalized Po versus QF curves (Fig. 1C) and the chlorophyll-normalized Po versus PAR curves (Fig. S1), which were consistent between both low and high light acclimated cultures. This efficiency across a range of photoacclimation conditions likely contributes to the ecological success of diatoms in dynamic light environments (Behrenfeld et al, 2021). quantum flux (QF) as the independent variable. The 1-qL versus QF curves (Fig. 1D), the shape of the

421 chlorophyll fluorescence parameters versus QF curves (Fig. $1 \mathrm{E}, \mathrm{F}$ ), and the D1 content as a fraction of 422 total protein (Table S1) were consistent between low and high light. Contrasting PAM vs. QF (Fig. 1E, F)

423 with PAM vs. PAR (Fig. S5A, B) illustrates how interpreting photophysiology from a QF perspective affects 424 conclusions about photophysiology. Using QF as the independent variable not only normalized the 425 comparisons across light regimes (fluorescent bulb for culturing, red LED for PAM experiments) and 
experimental apparatus (e.g., Roux flasks for culturing versus a round cuvette for PAM measurements),

427 this approach also accounted for inherent changes in photophysiology (e.g., pigment content, 428 photochemical efficiency).

We observed very little dissipation of excitation energy via NPQ at the experimental QF values (Fig. value of 0.32 (Table 2 ) suggesting the presence of alternative dissipative mechanisms [Y(NO)]. NPQ has been shown to be an important excitation energy dissipation mechanism in dynamic light conditions

433 (Olaizola et al, 1994; Lavaud et al, 2002; Wagner et al, 2006); however, our results suggest these other dissipative mechanisms are sufficient to prevent photoinhibition under stable light environments. Overall, these data suggest $P$. tricornutum employs a photoacclimation strategy that emphasizes rapid utilization and dissipation of light energy. This strategy results in conditions where the overall photosynthetic

437 apparatus is under-utilized, as in our low light acclimated cultures. However, our rapid-light-curve experiments suggest this allows $P$. tricornutum to immediately respond to an increase in available photon flux without the need to biosynthesize additional macromolecules, evident from consistency in total photosystem II content per cell and the redox state of the plastoquinone pool as a function of quantum flux (Fig. S2A, B). genome-scale model resulted in accurate predictions of photoautotrophic growth (Fig. 6). Growth rate in the $\mathrm{HL}$ condition was underestimated by $12 \%$; however, this was likely due to the beginning of carbon 445 limitation in the sample during short-term measurements of photosynthetic capacity (Fig. S3A). We chose 446 not to spike in exogenous bicarbonate for our $\mathrm{O}_{2}$-evolution measurements as we were interested in 447 measuring photosynthetic parameters relevant to our culturing conditions. However, our simulations 448 underestimated the growth rate at high light, suggesting the carbon environment needs be considered when performing rapid light curves (indeed, standard protocol is to include several $\mathrm{mM} \mathrm{NaHCO}_{3}{ }^{-}$in these 
assays to avoid carbon limitation). Our efforts to establish the hierarchy of constraints suggested photon uptake and chlorophyll fluorescence constraints alone accurately predict growth rate at low acclimation

452 irradiances (Table 3). This opens the possibility for non-invasive monitoring of culture health and productivity for biotechnology applications. Bypassing a portion of a production culture through a passive

454 sampling window with integrated chlorophyll fluorescence and spectral absorption analysis, coupled with 455 data on surface irradiance, would recreate the chlorophyll fluorescence and photon uptake constraints. As bioproduction conditions are usually high density, which would likely result in low cell-specific quantum

457 flux, integrating non-invasive sampling with our modeling construct could result in accurate measurements of photoautotrophic metabolism in these settings. parameters in a manner similar to previous investigations (Wagner et al, 2006; Jakob et al, 2007). However, GEMs also predict the optimal distribution of excitation energy between PSI and PSII, an advantage compared to previous work where it was assumed $50 \%$ of the absorbed quanta were directed to each photosystem. Our simulations predicted a two-fold increase excitation energy was utilized by PSII under

464 high light conditions compared to low light conditions, resulting in approximately $76 \%$ of absorbed quanta directed to PSII. However, there was a similar number of charge separation events at both photosystems

466 (Table 4). This is consistent with the observation that there is minimal cyclic electron flow (CEF) around 467 PSI in P. tricornutum (Bailleul et al, 2015) and energy dissipation of light energy at PSII Y(NO), which would 468 result in roughly equivalent charge separations at both photosystems. Our model derived ETR differs from 469 methodologies that assume equal excitation energy routed to both photosystems and a lower predicted 470 quantum demand at experimental QF values (Table 4). For an organism like $P$. tricornutum that does not 471 employ extensive CEF, the advantage of this approach is diminished. However, for microalgae that 472 dynamically reroute excitation energy between the photosystems and adjust their biomass 473 macromolecular composition as part of their photoacclimation strategy [e.g. Chlamydomonas reinhardtii 
474 (Davis et al, 2013; Lucker \& Kramer, 2013)], implicitly calculating excitation routing between

475 photosystems using our modeling framework will result in better approximations of ETR.

Incorporation of chlorophyll fluorescence measurements as a GEM constraint increased the accuracy

477 of model predictions. Accounting for photon loss upstream of the photosystems resulted in a more

478 accurate prediction of intracellular oxygen production and reductant-mediated oxygen consumption.

479 These new constraints affected predictions related to excess reductant in the system (EET) and cross-

481 correlated to NPQ activation (Fig. 1F), suggesting saturation of EET pathways triggers this photoprotective energy until the steady-state reduction of the plastoquinone pool is approximately $50 \%$. At this point, EET

485 is activated to facilitate re-oxidation of the photosynthetic electron transport chain. At a QF of approximately $0.6 \mathrm{fmol}$ photons cell $\mathrm{f} \mathrm{s}^{-1}$, the EET pathways are saturated, the steady-state reduction of the plastoquinone pool reaches $70 \%$, and NPQ is activated to assist in dissipating captured photons.

488 Therefore, the model accurately recreates the onset of NPQ that occurs when light absorption outpaces 489 the ability to utilize this energy within metabolism. Interestingly, this model is consistent for cells 490 acclimated to both low and high light, and Phaeodactylum is known to maintain high capacity for NPQ in 491 both light conditions (Taddei et al, 2018).

494 in this study is generally applicable to all phototrophic genome-scale model simulations and previous 495 efforts using experimentally-derived electron transport efficiencies, as opposed to PAM, showed good agreement with ${ }^{13} \mathrm{C}$ metabolic flux analysis (Broddrick et al, 2019b). 
The interest in integrating light-driven metabolism into bioengineering and synthetic biology necessitates an iterative framework for bioprocess development. The design-build-test-learn (DBTL) paradigm is one such approach that has been leveraged to rapidly increase bioproduct titers (Petzold et al, 2015; Carbonell et al, 2018). Computational tools are integral to these workflows. Relevant to the design step of the process, GEMs have been extensively used to rationally engineer metabolism to generate a wide variety of phenotypes (Czajka et al, 2021; Bang et al, 2020; Li et al, 2019). We explored approaches is underrepresented in phototrophic systems. Initial assessments quantified the reductant cost of cellular macromolecules, which provided insight into the theoretical yield of different compound pathway (chorismate), and isoprenoid precursors (isopentenyl pyrophosphate) were only partially fueled

513 by reductant that would normally be dissipated by EET, showing there is still considerable potential 514 associated with the engineering of primary photosynthetic metabolism to increase the overall yields of 515 bioproducts.

517 metabolic precursors of industrial interest may require significant engineering of central carbon 518 metabolism. For instance, increasing the production of our three selected metabolites increased the flux 519 of 3-phospho-D-glycerate (3pg) through the plastid glycolytic pathway (Figure 5). Additionally, the 520 increased flux of carbon to isoprenoid biosynthesis or through the shikimate pathway puts an increased 
521 demand for biosynthetic intermediates that are sourced from the CBBC (glyceralde-3-phosphate and D-

522 erythrose-4-phosphate, respectively). Photosynthetic microorganisms, which include diatoms, naturally

523 redirect carbon and energy to storage molecules under conditions of nutrient deprivation, such as

524 nitrogen limitation (Alipanah et al, 2015). This phenotype forms the basis for much of the interest in

525 biofuel applications of these phototrophs (Levering et al, 2015). Thus, a reasonable strategy and potential

526 future direction is the diel separation of carbon fixation and bioproduct formation. It may be that

527 rerouting these metabolites from sugar polymer degradation via the pentose phosphate pathway or

528 mitochondrial $\beta$-oxidation of lipids (Jallet et al, 2020) may alleviate some of the pressure on the CBBC.

529 The next step is to build and test these strains to initiate the first iteration of the DBTL cycle.

530 During the test phase, the model constraints provide a roadmap for relevant process parameters and a

531 framework to evaluate process performance, to include assessments on the efficiency of EET usage. It is

532 important to note our design simulations do not include possible changes in photophysiology due to strain

533 engineering (e.g., an increase in $\mathrm{P}_{\mathrm{o}}$ ). However, physiological outputs from the testing of the strain designs

534 proposed can be re-integrated into the modeling framework described here to include changes in

535 experimentally derived constraints. This contributes to the learn step of the DBTL cycle, closing the loop

536 on the first iteration and enabling an updated design strategy for the next iteration.

Taken together, our results show integrating relevant measurements of photosynthetic

538 physiology with genome-scale models results in quantitative predictions of condition-specific phenotypes.

539 This paves the way for iterative design and real-time process control of photobioproduction platforms. 


\section{Methods}

\section{Culture conditions}

Phaeodactylum tricornutum CCAP 1055/1 was grown axenically in silicon-free Instant Ocean

544 artificial seawater (salinity 35\%). Nutrients were added according to the stoichiometry described in

545 (Guillard, 1975) but at 2.3 fold higher concentrations to avoid nutrient limitation. Cells were cultured at $54618^{\circ} \mathrm{C}$ in $400 \mathrm{~mL}$ medium in $1 \mathrm{~L}$ Roux flasks. Flasks were bubbled with air $\left(1 \mathrm{~L}^{\text {air } \mathrm{L}^{-1} \text { culture minute }} \mathrm{e}^{-1}\right)$ under 547 continuous illumination in a temperature-controlled incubator. Cultures were light acclimated (low light $548(n=3)$ at $60 \mu \mathrm{mol}$ photons $\mathrm{m}^{-2} \mathrm{~s}^{-1}$, high light $(\mathrm{n}=4)$ at $600 \mu \mathrm{mol}$ photons $\left.\mathrm{m}^{-2} \mathrm{~s}^{-1}\right)$ for 72 hours, diluted and 549 grown until mid-exponential phase before being harvested.

\section{Cell physiology measurements}

Cell densities were determined using a BD Accuri C6 flow cytometer as described in (Jallet et al, 2016a). Growth rates were determined based on the change in cell counts from inoculation to harvest. overnight. Cellular dry weight was determined by subtracting the post-drying mass from the pre-drying mass, after normalizing to the media control.

\section{Determination of cell dimensions}

One $\mu \mathrm{L}$ of Lugol's solution was added to $1 \mathrm{~mL}$ of culture and the sample was stored at $4^{\circ} \mathrm{C}$ until analysis. For imaging, thin pads of $1 \%(w t / v o l)$ agarose were prepared using Mini-PROTEAN R Tetra Cell

$561 \mu \mathrm{l}$ cell culture liquid was added to the pad and let dry. Then a microscope slide cover was gently placed 562 onto of the agarose pad and cells were imaged using a DeltaVision inverted epi-fluorescence microscope 563 (Applied Precision, Issaquah, WA). Images were captured using a CoolSnap HD charge-coupled device 564 (CCD) camera (Photometrics, Tucson, AZ). Cell length and width were determined using the straight-line 
tool in ImageJ (Schindelin et al, 2015) and used to determine cell volume [high light ( $\mathrm{n=94}$ ) and low light

566 ( $n=46)$ acclimated cells]. P. tricornutum was modeled as a core ellipse with two cones extending away

567 from the core ellipse. The core ellipse was calculated according to the following equations:

568

569

570

571

572

573

574 575 determined using the Python Numpy package (Harris et al, 2020).

576

577

578

579

580

581

582

583

584

585

586

587

$$
\begin{array}{cc}
V o l=\frac{4}{3} \pi\left(S_{m a j} \times S_{\text {min }}^{2}\right) & \\
S_{m a j}=\frac{(l-2 w)}{2} & \text { Eq. } 2 \\
S_{\text {min }}=\frac{w}{2} & \text { Eq. } 3
\end{array}
$$

where I is the cell length and $w$ is the cell width.

The cones were calculated according to the following equation:

\section{Pigment extraction} were determined using the equations for methanol (Ritchie, 2008).

\section{Cellular absorption coefficients}$$
\text { Eq. } 1
$$

$$
V o l=\frac{1}{3} \pi\left(\left(r_{2}\right)^{2}+\left(r_{2} r_{1}\right)+\left(r_{1}\right)^{2}\right) h \quad \text { Eq. } 4
$$

where $r_{2}$ is equal to $w / 4, r_{1}$ is equal to $1 \mu \mathrm{m}$ and $\mathrm{h}$ the cell width. Mean and standard deviation were

Cells ( $4 \mathrm{~mL}$ of liquid culture) were collected by centrifugation at $10,000 \times \mathrm{g}$ at $5^{\circ} \mathrm{C}$ for 15 minutes. The supernatant was discarded and the cell pellet was frozen at $-80^{\circ} \mathrm{C}$ until processing. Chlorophyll was extracted with $50 \mu \mathrm{L}$ DMSO and $1950 \mu \mathrm{L}$ of methanol, incubated in the dark for 30 minutes, and centrifuged at $10,000 \times \mathrm{g}$ at $5^{\circ} \mathrm{C}$ for 15 minutes. The pigment containing supernatant was transferred to a $1 \mathrm{~cm}$ path length cuvette. Absorbance spectra were collected using a Cary 60 UV-Vis Agilent spectrophotometer in scan mode ( $350-800 \mathrm{~nm}$, scan interval of $1 \mathrm{~nm})$. Chlorophyll $a$ and $c$ concentrations

Cellular absorption coefficients were determined based on published protocols (Moore et al, 1995). Approximately $5 \times 10^{7}$ cells were collected by filtration onto a GF/A glass microfiber filter (21 mm diameter). The filter was cut to fit in a $1 \mathrm{~cm}$ path length cuvette and placed against the inside of the 

averaged. To decrease the noise from filter scattering, the absorbance spectra were smoothed using a amplification according to the following equation:

$$
a_{\lambda}=2.303\left(A\left(O D_{\lambda}\right)+B\left(O D_{\lambda}^{2}\right)\right)
$$

Eq. 5

where $O D_{\lambda}$ is the absorbance at a given wavelength and $A$ and $B$ are species-specific coefficients for the

deposited ( $2.1 \mathrm{~cm}^{2}$ for the $21 \mathrm{~mm}$ diameter GF/A filter).

\section{Simultaneous oxygen evolution and chlorophyll fluorescence parameters}

605 FireSting Optical Oxygen Meter were used for the simultaneous measurement of chlorophyll fluorescence 606 and oxygen evolution. Approximately $30 \mathrm{~mL}$ of culture was removed, and cells were pelleted by 607 centrifugation ( $3000 \times \mathrm{g}, 10$ minutes at the experimental temperature). Cell pellets were resuspended in 608 fresh media to the target cell density ( $\mathrm{HL}: 2 \times 10^{7}$ cells $\mathrm{mL}^{-1}, \mathrm{LL}: 1 \times 10^{7}$ cells $\mathrm{mL}^{-1}$ ) and kept in the dark for 10 609 minutes prior to analysis. For select experiments, the cells were reconstituted in fresh media 610 supplemented with $5 \mathrm{mM}$ sodium bicarbonate. Dark respiration rates were collected for approximately 
612

613

614

615

616

617

618

619

620

621

622

623

624

625

626

627

628

629

630

631

632

633

634

635

$\mathrm{ms}, 10,000 \mu \mathrm{mol}$ photons $\mathrm{m}^{-2} \mathrm{~s}^{-1}$ ) for fluorescence measurements. Cells were step illuminated (HL: 60 seconds, LL: 90 seconds) at the following increasing intensities ( $\mu \mathrm{mol}$ photons $\left.\mathrm{m}^{-2} \mathrm{~s}^{-1}\right)$ :

$\mathrm{HL}: 0,8,24,43,75,109,146,195,259,339,435,547,674,844,1033,1565,2386,2924$

LL: $0,8,24,43,75,109,146,195,259,339,435,547,844,1565,2386$,

followed by the red actinic saturating pulse.

The chlorophyll fluorescence parameters $F_{v} / F_{m}, Y(I I), 1-q L$ and NPQ were determined as described

(Schreiber et al, 1995; Kramer et al, 2004). Shading in the round cuvette was accounted for by calculating the attenuation across the cuvette path length as described previously (Broddrick et al, 2019b):

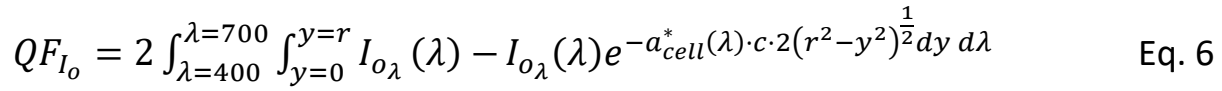

where $Q F_{10}$ is the quantum flux in $\mu$ mol photons $m^{-2} s^{-1}$ at a given PAR value $\left(I_{0}\right), \lambda$ is the wavelength, $I_{\lambda}$

$(\lambda)$ is the fraction of the PAR at a given wavelength $\lambda, r$ is the radius of the cuvette $(0.56 \mathrm{~cm})$, a* cell is the wavelength-specific absorption coefficient in $\mathrm{cm}^{2}$ cell $^{-1}$, and $\mathrm{c}$ is the cell density in cells $\mathrm{cm}^{-3}$. QF was converted to $\mu \mathrm{mol}$ photons cell ${ }^{-1} \mathrm{~s}^{-1}$ by multiplying $\mathrm{QF}_{\mathrm{lo}}$ by the rectangular surface area of the cuvette (width $=0.56 \mathrm{~cm}$, height $=1.15 \mathrm{~cm}$ ), converted to $\mathrm{m}^{2}$ and divided by the total number of cells in the cuvette. This QF value was used as the independent variable in plots of oxygen-based photosynthesis $\left(\mathrm{P}_{0}\right)$ versus QF.

Time-course measurements of oxygen evolution were exported from the FireSting O2 Logger software as a .txt file. The data was aligned to the PAM data irradiance values and the first 10 seconds of data after each increase in irradiance was discarded as the $\mathrm{O}_{2}$ evolution rate stabilized at the new irradiance value. The oxygen evolution rate was determined by taking the slope of the $\mathrm{O}_{2}$ versus time plot for the remaining time interval ( 50 seconds for $\mathrm{HL}, 80$ seconds for $\mathrm{LL}$ ) using the Python SciPy package (scipy.stats.linregress) (Virtanen et al, 2020). The resulting oxygen evolution rates were then normalized to cell counts. Dark period respiration rates were determined in a similar manner by taking the slope of the oxygen consumption versus time curve for the last 2 minutes of the 10-minute dark acclimation period. 


\section{Membrane inlet mass spectrometry (MIMS)}

Membrane inlet mass spectrometry was measured similarly to (Broddrick et al, 2019a) and (Ware et al, 2020). Cells were collected for chlorophyll quantification according to (Jallet et al, 2016a), with spectrophotometric quantification performed in $100 \%$ methanol according to the equations provided in (Ritchie, 2008). Samples corresponding to $4 \mu \mathrm{g}$ chlorophyll $a \mathrm{~mL}^{-1}$ were collected and resuspended in fresh $\mathrm{f} / 2$ media. All steps were performed at $18^{\circ} \mathrm{C} .2 \mathrm{~mL}$ of culture was loaded into a custom made cylindrical Accura ClearVue SLA cuvette. Oxygen evolution and consumption was measured using a quad mass spectrometer (Pfeiffer PrismaPlus QMG220, Quadera v4.62). Dissolved gas was pulled through a silicon membrane, connected to the mass spectrometer via a stainless-steel tube with a vapor trap (ethanol and dry ice). Cells were bubbled with $\mathrm{N}_{2}$ to deplete oxygen $\left({ }^{16} \mathrm{O}_{2}, \mathrm{~m} / \mathrm{z}=32\right)$ from the suspension to approximately $50 \%$ of atmospheric oxygen concentrations. Cells were kept under constant stirring via a magnetic stir bar. The suspensions were injected with ${ }^{18} \mathrm{O}_{2}$ (Cat \#490474, Aldrich) and mixed for $10-15$ minutes until equilibration was achieved. ${ }^{18} \mathrm{O}_{2}$ was then purged from the sample using a stopper leaving a $1.4 \mathrm{~mL}$ final culture volume. Gas consumption was measured in the dark for 5 minutes to calculate the respiration rate. Cells were then illuminated with a blue measuring light (Walz, Dual-PAM-100) to achieve a $0.2 \mathrm{~V}$ fluorescence signal for 15 minutes to relax photoprotective processes. A white LED programmed to achieve 600 or $60 \mu \mathrm{mol}$ photons $\mathrm{m}^{-2} \mathrm{~s}^{-1}$ (measured with Walz, ULM-500, US-SQS/L attachment) was used to illuminate cultures to their corresponding in situ light intensity for 7 minutes. The slopes of oxygen consumption $\left({ }^{16} \mathrm{O}_{2}, \mathrm{~m} / \mathrm{z}=32\right.$, and $\left.{ }^{18} \mathrm{O}_{2}, \mathrm{~m} / \mathrm{z}=36\right)$ and evolution $\left({ }^{16} \mathrm{O}_{2}, \mathrm{~m} / \mathrm{z}=32\right)$ were calculated according to (Beckmann et al, 2009) using the mass charge $(\mathrm{m} / \mathrm{z})$ change on a per second basis, calculated over the last 30 s of illumination. Argon $(\mathrm{m} / \mathrm{z}=40)$ was used to normalize oxygen concentrations, minimizing the effects of pressure change and abiotic gas consumption (Bailleul et al, 2015).

\section{Genome-scale metabolic modeling}


660 (Broddrick et al, 2019a). Simulations were performed in a similar manner to (Broddrick et al, 2019b). The biomass objective function was updated to account for differences in pigments between the low and high coming from the side of the flask. The Roux flasks had approximately $375 \mathrm{~mL}$ of culture at the time of the

$$
I_{a}=\int_{400}^{700} I_{o}(\lambda)-I_{o}(\lambda) e^{-a_{c e l l}^{*}(\lambda) * \frac{c e l l}{m L} * l} d \lambda
$$

673 The sum of absorbed light at each wavelength between 400 and $700 \mathrm{~nm}$ was used to set the reaction

674 bounds of the photon exchange reactions in the GEM (reaction ID: EX_photon_e). quantum flux as the independent variable.

$$
P=P_{\max }\left(1-e^{-\frac{\alpha \times Q F}{P_{\max }}}\right) e^{-\frac{\beta \times Q F}{P_{\max }}} \quad \text { Eq. } 8
$$

$678 P_{\max }$ is the maximum photosynthetic rate, and $\alpha$ and $\beta$ are parameters that describe the initial slope of the 679 curve, and the photoinhibition (if present), respectively. The respiration rate was added to all values prior to generating the fit since the Platt curve is forced through the origin. The respiration rate was then

681 subtracted from the fit to return the curve to the gross oxygen evolution rate. These curves were used to 
determine the oxygen evolution rate based on the total absorbed quantum flux and used to set the

683 bounds of the oxygen exchange reaction in the GEM (reaction ID: EX_o2_e).

684

Integrating chlorophyll fluorescence measurements into the genome-scale model: Chlorophyll

685

fluorescence parameters were incorporated into the GEM based on the experimental values for Y(II),

$686 \mathrm{Y}(\mathrm{NPQ})$ and $\mathrm{Y}(\mathrm{NO})$. The $\mathrm{Y}(\mathrm{II})$ vs. QF data was fit to an exponential decay function $(\mathrm{HL}: Y(I I)=$

687

$\left.0.64 e^{-1.17 \times 10^{9} * Q F}, \mathrm{R}^{2}=0.99 ; \mathrm{LL}: Y(I I)=0.70 e^{-8.54 \times 10^{8} * Q F}, \mathrm{R}^{2}=0.99\right) . \mathrm{Y}(\mathrm{NPQ})$ vs. QF was fit to a Hill

688 function:

689

$$
Y(N P Q)=\frac{1}{1+\left(\frac{K a}{Q F}\right)^{n}} \quad \text { Eq. } 9
$$

690

Where $\mathrm{HL}: \mathrm{n}=5.5, \mathrm{~K}_{\mathrm{a}}=1.37 \times 10^{-9}$ and $\mathrm{LL}: \mathrm{n}=3, \mathrm{~K}_{\mathrm{a}}=2.30 \times 10^{-9}$. For each simulation, the calculated QF was used to determine the $\mathrm{Y}(\mathrm{II})$ and $\mathrm{Y}(\mathrm{NPQ})$ values. $\mathrm{Y}(\mathrm{NO})$ was defined as:

$$
Y(N O)=1-\left(1-\frac{F_{v}}{F_{m}}\right)-Y(I I)-Y(N P Q) \quad \text { Eq. } 10
$$

The fraction of absorbed photons available to perform photochemistry was constrained using the

694 following model reaction:

$$
P H O \_P S I I t u: p h o t o n \_h \rightarrow A \text { photon_1 }-F v F m_{-} u+B \text { photon_YII_u }+C \text { photon_YNPQ_u }+D \text { photon_YNO_u Eq. } 10
$$

696 Where PHO_PSIIt_u is the model reaction name, photon_h is the pool of excitation energy available to 697 both PSII and PSI to perform photochemistry (equivalent to quantum flux, QF), $A$ is the fraction of excitation energy lost upstream of the photosystems, B is the $\mathrm{Y}(\mathrm{II})$ value at the experimental $\mathrm{QF}, \mathrm{C}$ is the Y(NPQ) at the experimental QF, and D is $Y(N O)$ at the experimental QF. Only photon_YII_u was included

700 in the model PSII reaction; thus, limiting the amount of excitation energy to perform photochemistry to 701 the $Y(I I)$ fraction. 
which forces a minimal amount of reductant mediated oxygen consumption consistent with the observed dark respiration rate.

above. Exponential phase cultures were split into control and lincomycin treatments in biological triplicates. Lincomycin infiltration was achieved by inoculating cultures with $500 \mu \mathrm{g} \mathrm{mL}^{-1}$ lincomycin for 10 minutes in the dark (Key et al, 2010). Samples were collected for cell counts, absorption spectra, $F_{v} / F_{m}$ transferred to $10 \mu \mathrm{mol}$ photons $\mathrm{m}^{-2} \mathrm{~s}^{-1}$ with a red-light filter for 20 minutes to relax NPQ. Cells were collected on a glass fiber prefilter (Merck Millipore Ltd). A measuring light intensity was applied to elicit a determination of $F_{0}$. A saturating pulse $\left(600 \mathrm{~ms}, 10,000 \mu \mathrm{mol}\right.$ photons $\left.\mathrm{m}^{-2} \mathrm{~s}^{-1}, 635 \mathrm{~nm}\right)$ was applied to

715 determine $F_{m}$, and calculate the maximum photochemical quantum yield of $P S I I\left(F_{v} / F_{m}\right)$. The D1 concentration as a fraction of total protein was determined by SDS-PAGE and Western blots as detailed in (Jallet et al, 2016a) with some minor modifications. For Western blot analysis, $0.8 \mu \mathrm{g}$ of total sample 718 protein in $25 \mu$ total volume was loaded into Novex WedgeWell $10-20 \%$ Tris-Glycine Gels. Alongside samples, PsbA D1 protein quantitation standards (Agrisera, \#AS01 016S) were run. Standards were loaded at the same volume as samples, being diluted in the same sample buffer (Jallet et al, 2016a). D1 protein

721 standards were loaded at three concentrations, ensuring samples fell within linear range. Standards and 722 samples D1 protein content was quantified by densitometry (ImageJ, v1.53 723 https://imagej.nih.gov/ij/download.html). Values from the quantification of sample D1 protein 724 concentration were divided by total protein loaded to calculate D1 protein as a percentage of total protein. 725 The D1 decay rate in lincomycin treated cells was determined using equation 5 from (Campbell \& 726 Tyystjärvi, 2012). 
The first-order decay constant, $\mathrm{k}_{\mathrm{PI}}$ was calculated from the from the change in the maximum quantum yield of PSII $\left(F_{v} / F_{m}\right)$ between cells with and without lincomycin treatment. $[A]_{0}$ was determined from the D1 content as a fraction of total protein calculated from the Western blot analysis. The steady-state D1 damage rate in percent total protein $\mathrm{h}^{-1}$ is equal to the first derivative of the above equation solved at a given steady-state fraction of D1 subunits undergoing repair:

$$
D 1_{\text {damage }}=-k_{P I}[A]_{0} e^{-k_{P I} t} \quad \text { Eq. } 12
$$

We approximated the steady-state fraction undergoing repair using the difference between $F_{v} / F_{m}$ and $Y(I I)$ values in the RLCs with a $10 \mathrm{~s}$ blue light treatment, at the experimental QF (Fig. S4), under the hypothesis the residual decrease in quantum efficiency was due to inactive PSII complexes undergoing repair. The final D1 damage constraint in mmol D1 $\mathrm{gDW}^{-1} \mathrm{~h}^{-1}$ was calculated with the following equation:

$$
N G A M_{-} D 1=\frac{g D 1}{g \text { protein } \times \text { hour }} \times \frac{g \text { protein }}{g D W} \times \frac{1 \text { mmol } D 1}{39.651 g D 1} \quad \text { Eq. } 13
$$

Where the first term is the steady-state damage rate, the second term is the protein fraction of the total biomass ( 0.70 for high light, 0.64 for low light) and the third term is the formula mass of the $P$. tricornutum D1 subunit $(39,651 \mathrm{Da})$. The model constraint was set from the mean steady-state damage rate and the upper and lower bounds were calculated by combining the variances of \pm 1 percent standard deviation of the Western blot analysis, \pm 1 percent standard deviation of the $k_{P I}$ value from the lincomycin treatment data, and a $5 \%$ error on the biomass protein fraction for a total of $20 \%$ standard deviation for the high light value and a $29 \%$ standard deviation for the low light value.

Genome-scale model simulations: The simulation was performed by maximizing the biomass objective function (BOF) reaction using the parsimonious FBA function (Lewis et al, 2010) as implemented in COBRApy (Ebrahim et al, 2013). The flux through this reaction is equal to the biomass accumulation in milligrams over the 20-minute time interval. This biomass was added to a running total of the total culture biomass and used to parameterize the next 20-minute simulation interval. The simulations included the mean and \pm 1 standard deviation of the $a{ }^{*}$ cell values, cell dry weight to determine the cell count at each 
752 time interval, and the oxygen evolution rate versus QF. This resulted in a set of 27 parameters for which

753 growth rate was determined using the following equation:

$$
\mu=\frac{\ln \left(\frac{N_{t}}{N_{0}}\right)}{t} \quad \text { Eq. } 14
$$

The mean, maximum and minimum growth rates are reported. For the bicarbonate spiked simulation, the mean values were used for all parameters except the oxygen uptake rate which was parameterized using a Platt fit through the bicarbonate spiked $P_{0}$ vs. QF curve. Reaction fluxes were exported into a csv file with Python and visualized in Escher (King et al, 2015). following constraints (unconstrained refers to an arbitrarily high value that doesn't limit growth): photons $(h v)$ : the model photon exchange reaction (EX_photon_e) was set to the calculated QF value; oxygen evolution $\left(\mathrm{P}_{\mathrm{O}}\right)$ : the model reaction EX_02_e lower bound was set to the experimental oxygen evolution rate at the calculated QF; D1: the D1 damage rate was included; Y(II): the pseudo-reaction PHO_PSIIt_u that accounts for the $1-F_{v} / F_{m}, Y(I I), Y(N P Q)$ and $Y(N O)$ fractions was included. To calculate excess energy in the system a demand reaction was added to the model upstream of the photosystems that allowed any excess QF to leave the model (reaction ID: DM_photon_c). The flux through this reaction is equal to the excitation energy in excess of the requirements to generate biomass and satisfy NGAM (see above). For all other simulations, the bounds of this reaction were set to 0 . For analyses that required a range of

769 feasible fluxes through a model reaction, flux variability analysis (FVA) was used as implemented in COBRApy (Ebrahim et al, 2013) with the 'loopless' option set to 'True'.

772 through photosystem I (PSI) minus the flux through cyclic electron flow (CEF). To determine the \%LET

773 allocated to each biomass component, we first determined the EET in the system by opening the 774 DM_photon_c reaction, set the lower bound of biomass objective function (BOF) to 99.9\% of maximum 775 and then maximized the DM_photon_c flux. This set the baseline LET required for generating biomass. 
776

777

778

779

We then iterated over each biomass component, removing the component from the BOF and then adjusting the BOF reaction bounds to (1-biomass component fraction) ${ }^{*}$ maximum. Flux through the DM_photon_c reaction was once again maximized and the difference between this flux value and that for the full BOF was considered the photons necessary to produce the removed biomass component.

Bioproduction of representative compounds were performed by selecting a model metabolite for biosynthesis (plastid fatty acids: hexadecanoate, model id: hdca_h; the shikimate pathway: chorismate, model id: chor_h; and isoprenoid precursors: isopentenyl pyrophosphate, model id: ipdp_h). The default model was solved for maximum biomass (in mg cell dry weight), and subsequent simulations fixed the biomass production at $10 \%$ intervals from $50 \%$ to $90 \%$ of this maximum. A demand reaction was added to the model allowing the representative pathway metabolite (hdca_h, chor_h, or ipdp_h) to leave the system. The model objective was set to maximize this demand reaction. EET was determined as outlined above for all combinations of biomass reallocation and metabolite production. All calculations and simulations were performed using in-house scripts developed in IPython (Fernando et al, 2007). For pathway engineering analysis, the reaction fluxes for the $70 \%$ biomass results ( $30 \%$ of cellular biomass rerouted to bioproduct formation) were exported into a csv file with Python and visualized in Escher (King et al, 2015). All simulation code, models, flux simulations and metabolic maps are available in the Supplemental Material.

\section{Data Availability}

All code used to analyze and generate the results and figures for this study, along with input data, can be found at https://github.com/JaredTBrod/PAM_GEMs.

\section{Acknowledgements}

The authors would like to thank Dr. David G. Welkie and Prof. Susan S. Golden at UC San Diego for their assistance in imaging $P$. tricornutum for the cell size measurements, as well as Marc Abrams for his critical review of the manuscript. 


\section{Conflict of Interests Statement}

The authors report no conflict of interests.

\section{References}

Alipanah L, Rohloff J, Winge P, Bones AM \& Brembu T (2015) Whole-cell response to nitrogen deprivation in the diatom Phaeodactylum tricornutum. J Exp Bot 66: 6281-6296

Bailleul B, Berne N, Murik O, Petroutsos D, Prihoda J, Tanaka A, Villanova V, Bligny R, Flori S, Falconet D, et al (2015) Energetic coupling between plastids and mitochondria drives $\mathrm{CO} 2$ assimilation in diatoms. Nature 524: 366-369

Bang J, Hwang CH, Ahn JH, Lee JA \& Lee SY (2020) Escherichia coli is engineered to grow on CO 2 and formic acid. Nat Microbiol 5: 1459-1463

Beckmann K, Messinger J, Badger MR, Wydrzynski T \& Hillier W (2009) On-line mass spectrometry: membrane inlet sampling. Photosynth Res 102: 511-522

Behrenfeld MJ, Halsey KH, Boss E, Karp-Boss L, Milligan AJ \& Peers G (2021) Thoughts on the evolution and ecological niche of diatoms. Ecol Monogr 91: e01457

Brey LF, Włodarczyk AJ, Bang Thøfner JF, Burow M, Crocoll C, Nielsen I, Zygadlo Nielsen AJ \& Jensen PE (2020) Metabolic engineering of Synechocystis sp. PCC 6803 for the production of aromatic amino acids and derived phenylpropanoids. Metab Eng 57: 129-139

Broddrick JT, Du N, Smith SR, Tsuji Y, Jallet D, Ware MA, Peers G, Matsuda Y, Dupont CL, Mitchell BG, et al (2019a) Cross-compartment metabolic coupling enables flexible photoprotective mechanisms in the diatom Phaeodactylum tricornutum. New Phytol 222: 1364-1379

Broddrick JT, Rubin BE, Welkie DG, Du N, Mih N, Diamond S, Lee JJ, Golden SS \& Palsson BO (2016) Unique attributes of cyanobacterial metabolism revealed by improved genome-scale metabolic modeling and essential gene analysis. Proc Natl Acad Sci 113: E8344-E8353

Broddrick JT, Welkie DG, Jallet D, Golden SS, Peers G \& Palsson BO (2019b) Predicting the metabolic capabilities of Synechococcus elongatus PCC 7942 adapted to different light regimes. Metab Eng 52: 42-56

Campbell DA \& Tyystjärvi E (2012) Parameterization of photosystem II photoinactivation and repair. Biochim Biophys Acta 1817: 258-265

Carbonell P, Jervis AJ, Robinson CJ, Yan C, Dunstan M, Swainston N, Vinaixa M, Hollywood KA, Currin A, Rattray NJW, et al (2018) An automated Design-Build-Test-Learn pipeline for enhanced microbial production of fine chemicals. Commun Biol 1: 1-10 
Chang RL, Ghamsari L, Manichaikul A, Hom EFY, Balaji S, Fu W, Shen Y, Hao T, Palsson B $\varnothing$, Salehi-Ashtiani $\mathrm{K}$, et al (2011) Metabolic network reconstruction of Chlamydomonas offers insight into lightdriven algal metabolism. Mol Syst Biol 7: 518

Czajka JJ, Oyetunde T \& Tang YJ (2021) Integrated knowledge mining, genome-scale modeling, and machine learning for predicting Yarrowia lipolytica bioproduction. Metab Eng 67: 227-236

Davis MC, Fiehn O \& Durnford DG (2013) Metabolic acclimation to excess light intensity in Chlamydomonas reinhardtii. Plant Cell Environ 36: 1391-1405

Dietz K-J, Turkan I \& Krieger-Liszkay A (2016) Redox- and Reactive Oxygen Species-Dependent Signaling into and out of the Photosynthesizing Chloroplast. Plant Physiol 171: 1541-1550

Dinh HV, King ZA, Palsson BO \& Feist AM (2018) Identification of growth-coupled production strains considering protein costs and kinetic variability. Metab Eng Commun 7: e00080

Ebrahim A, Lerman JA, Palsson BO \& Hyduke DR (2013) COBRApy: COnstraints-Based Reconstruction and Analysis for Python. BMC Syst Biol 7: 74

Edelman M \& Mattoo AK (2008) D1-protein dynamics in photosystem II: the lingering enigma. Photosynth Res 98: 609-620

Falkowski PG \& Owens TG (1980) Light-Shade Adaptation : TWO STRATEGIES IN MARINE PHYTOPLANKTON. Plant Physiol 66: 592-5

Fernando P rez, Pérez F, Granger BE \& Fernando P rez (2007) IPython: A System for Interactive Scientific Computing. Comput Sci Eng 9: 21-29

Finkel Z V. \& Irwin AJ (2001) Light absorption by phytoplankton and the filter amplification correction: cell size and species effects. J Exp Mar Biol Ecol 259: 51-61

Giovagnetti V, Ware MA \& Ruban AV (2015) Assessment of the impact of photosystem I chlorophyll fluorescence on the pulse-amplitude modulated quenching analysis in leaves of Arabidopsis thaliana. Photosynth Res 125: 179-189

Guillard RRL (1975) Culture of Phytoplankton for Feeding Marine Invertebrates. In Culture of Marine Invertebrate Animals pp 29-60. Boston, MA: Springer US

Harris CR, Millman KJ, van der Walt SJ, Gommers R, Virtanen P, Cournapeau D, Wieser E, Taylor J, Berg S, Smith NJ, et al (2020) Array programming with NumPy. Nature 585: 357-362

Jakob T, Wagner H, Stehfest K \& Wilhelm C (2007) A complete energy balance from photons to new biomass reveals a light- and nutrient-dependent variability in the metabolic costs of carbon assimilation. J Exp Bot 58: 2101-2112

Jallet D, Caballero MA, Gallina AA, Youngblood M \& Peers G (2016a) Photosynthetic physiology and biomass partitioning in the model diatom Phaeodactylum tricornutum grown in a sinusoidal light regime. Algal Res 18: 51-60 
Jallet D, Cantrell M \& Peers G (2016b) New players for photoprotection and light acclimation. In Chloroplasts: Current Research and Future Trends p np. Caister Academic Press

Jallet D, Xing D, Hughes A, Moosburner M, Simmons MP, Allen AE \& Peers G (2020) Mitochondrial fatty acid $\beta$-oxidation is required for storage-lipid catabolism in a marine diatom. New Phytol 228: 946-958

Key T, McCarthy A, Campbell DA, Six C, Roy S \& Finkel ZV (2010) Cell size trade-offs govern light exploitation strategies in marine phytoplankton. Environ Microbiol 12: 95-104

King ZA, Dräger A, Ebrahim A, Sonnenschein N, Lewis NE \& Palsson BO (2015) Escher: A Web Application for Building, Sharing, and Embedding Data-Rich Visualizations of Biological Pathways. PLOS Comput Biol 11: e1004321

King ZA, Lu J, Dräger A, Miller P, Federowicz S, Lerman JA, Ebrahim A, Palsson BO \& Lewis NE (2016) BiGG Models: A platform for integrating, standardizing and sharing genome-scale models. Nucleic Acids Res 44: D515-D522

Kramer DM, Johnson G, Kiirats O \& Edwards GE (2004) New Fluorescence Parameters for the Determination of $Q_{A}$ Redox State and Excitation Energy Fluxes. Photosynth Res 79: 209-218

Krause GH \& Weis E (1991) Chlorophyll Fluorescence and Photosynthesis: The Basics. Annu Rev Plant Physiol Plant Mol Biol 42: 313-349

Kumar G, Shekh A, Jakhu S, Sharma Y, Kapoor R \& Sharma TR (2020) Bioengineering of Microalgae: Recent Advances, Perspectives, and Regulatory Challenges for Industrial Application. Front Bioeng Biotechnol 8: 914

Lassen LM, Nielsen AZ, Ziersen B, Gnanasekaran T, Møller BL \& Jensen PE (2014) Redirecting Photosynthetic Electron Flow into Light-Driven Synthesis of Alternative Products Including HighValue Bioactive Natural Compounds. ACS Synth Biol 3: 1-12

Lavaud J, Rousseau B, van Gorkom HJ \& Etienne A-L (2002) Influence of the Diadinoxanthin Pool Size on Photoprotection in the Marine Planktonic Diatom Phaeodactylum tricornutum. Plant Physiol 129: $1398-1406$

Levering J, Broddrick J, Dupont CL, Peers G, Beeri K, Mayers J, Gallina AA, Allen AE, Palsson BO \& Zengler $\mathrm{K}$ (2016) Genome-scale model reveals metabolic basis of biomass partitioning in a model diatom. PLOS ONE 11: e0155038

Levering J, Broddrick J \& Zengler K (2015) Engineering of oleaginous organisms for lipid production. Curr Opin Biotechnol 36: 32-39

Lewis NE, Hixson KK, Conrad TM, Lerman JA, Charusanti P, Polpitiya AD, Adkins JN, Schramm G, Purvine SO, Lopez-Ferrer D, et al (2010) Omic data from evolved E. coli are consistent with computed optimal growth from genome-scale models. Mol Syst Biol 6: 390 
Li C-T, Yelsky J, Chen Y, Zuñiga C, Eng R, Jiang L, Shapiro A, Huang K-W, Zengler K \& Betenbaugh MJ (2019) Utilizing genome-scale models to optimize nutrient supply for sustained algal growth and lipid productivity. Npj Syst Biol App/ 5: 1-11

Lucker B \& Kramer DM (2013) Regulation of cyclic electron flow in Chlamydomonas reinhardtii under fluctuating carbon availability. Photosynth Res 117: 449-459

Moore LR, Goericke R \& Chisholm SW (1995) Comparative physiology of Synechococcus and Prochlorococcus: influence of light and temperature on growth, pigments, fluorescence and absorptive properties. Mar Ecol Prog Ser 116: 259-275

Murik O, Tirichine L, Prihoda J, Thomas Y, Araújo WL, Allen AE, Fernie AR \& Bowler C (2019) Downregulation of mitochondrial alternative oxidase affects chloroplast function, redox status and stress response in a marine diatom. New Phytol 221: 1303-1316

Nicol L, Nawrocki WJ \& Croce R (2019) Disentangling the sites of non-photochemical quenching in vascular plants. Nat Plants 5: 1177-1183

Nixon PJ, Barker M, Boehm M, de Vries R \& Komenda J (2005) FtsH-mediated repair of the photosystem II complex in response to light stress. J Exp Bot 56: 357-363

Niyogi KK (2000) Safety valves for photosynthesis. Curr Opin Plant Biol 3: 455-460

Nymark M, Valle KC, Brembu T, Hancke K, Winge P, Andresen K, Johnsen G \& Bones AM (2009) An integrated analysis of molecular acclimation to high light in the marine diatom $<i>$ Phaeodactylum tricornutum<i/>. PLOS ONE 4: e7743

O’Brien EJ, Lerman JA, Chang RL, Hyduke DR, Palsson B $\emptyset$, Adadi R, Volkmer B, Milo R, Heinemann M, Shlomi T, et al (2013) Genome-scale models of metabolism and gene expression extend and refine growth phenotype prediction. Mol Syst Biol 9: 693

Olaizola M, La Roche J, Kolber Z \& Falkowski PG (1994) Non-photochemical fluorescence quenching and the diadinoxanthin cycle in a marine diatom. Photosynth Res 41: 357-370

Peers G (2014) Increasing algal photosynthetic productivity by integrating ecophysiology with systems biology. Trends Biotechnol 32: 551-555

Petzold C, Chan L, Nhan M \& Adams P (2015) Analytics for metabolic engineering. Front Bioeng Biotechnol 3: 135

Pfündel EE, Klughammer C, Meister A \& Cerovic ZG (2013) Deriving fluorometer-specific values of relative PSI fluorescence intensity from quenching of F0 fluorescence in leaves of Arabidopsis thaliana and Zea mays. Photosynth Res 114: 189-206

Ritchie RJ (2008) Universal chlorophyll equations for estimating chlorophylls $a, b, c$, and d and total chlorophylls in natural assemblages of photosynthetic organisms using acetone, methanol, or ethanol solvents. Photosynthetica 46: 115-126 
Santos-Merino M, Torrado A, Davis GA, Röttig A, Bibby TS, Kramer DM \& Ducat DC (2021) Improved photosynthetic capacity and photosystem I oxidation via heterologous metabolism engineering in cyanobacteria. Proc Natl Acad Sci 118: e2021523118

Schindelin J, Rueden CT, Hiner MC \& Eliceiri KW (2015) The ImageJ ecosystem: An open platform for biomedical image analysis. Mol Reprod Dev 82: 518-529

Schreiber U, Bilger W \& Neubauer C (1995) Chlorophyll Fluorescence as a Nonintrusive Indicator for Rapid Assessment of In Vivo Photosynthesis. In Ecophysiology of Photosynthesis pp 49-70. Berlin, Heidelberg: Springer Berlin Heidelberg

Taddei L, Chukhutsina VU, Lepetit B, Stella GR, Bassi R, van Amerongen H, Bouly J-P, Jaubert M, Finazzi G \& Falciatore A (2018) Dynamic Changes between Two LHCX-Related Energy Quenching Sites Control Diatom Photoacclimation. Plant Physiol 177: 953-965

Virtanen P, Gommers R, Oliphant TE, Haberland M, Reddy T, Cournapeau D, Burovski E, Peterson P, Weckesser W, Bright J, et al (2020) SciPy 1.0: fundamental algorithms for scientific computing in Python. Nat Methods 17: 261-272

Wagner H, Jakob T \& Wilhelm C (2006) Balancing the energy flow from captured light to biomass under fluctuating light conditions. New Phytol 169: 95-108

Ware MA, Hunstiger D, Cantrell M \& Peers G (2020) A Chlorophyte Alga Utilizes Alternative Electron Transport for Primary Photoprotection. Plant Physiol 183: 1735-1748

Zuñiga C, Levering J, Antoniewicz MR, Guarnieri MT, Betenbaugh MJ \& Zengler K (2017) Predicting dynamic metabolic demands in the photosynthetic eukaryote Chlorella vulgaris. Plant Physiol: 17006051-6052017 


\begin{tabular}{|c|c|c|c|c|}
\hline & $\begin{array}{l}\text { D1 damage } \\
\text { constant } \\
\left(\mathrm{min}^{-1}\right)\end{array}$ & $\begin{array}{c}\text { D1 protein } \\
\text { (\% total protein) }\end{array}$ & $\begin{array}{c}\text { D1 damage } \\
\text { constraint }^{*} \\
\left(\mathrm{mmol} \mathrm{D1} \mathrm{gDW}^{-1} \mathrm{~h}^{-1}\right)\end{array}$ & $\begin{array}{l}\text { Maintenance cost } \\
\left(\text { mmol NTP gDW }{ }^{-1} \mathrm{~h}^{-1}\right)\end{array}$ \\
\hline Low Light & $6 \pm 1 \times 10^{-4}$ & $1.1 \pm 0.2$ & $7 \pm 2 \times 10^{-6}$ & $8 \pm 2 \times 10^{-3}$ \\
\hline High Light & $2.33 \pm 0.17 \times 10^{-2}$ & $1.1 \pm 0.1$ & $2.53 \pm 0.51 \times 10^{-4}$ & $0.27 \pm 0.06$ \\
\hline
\end{tabular}

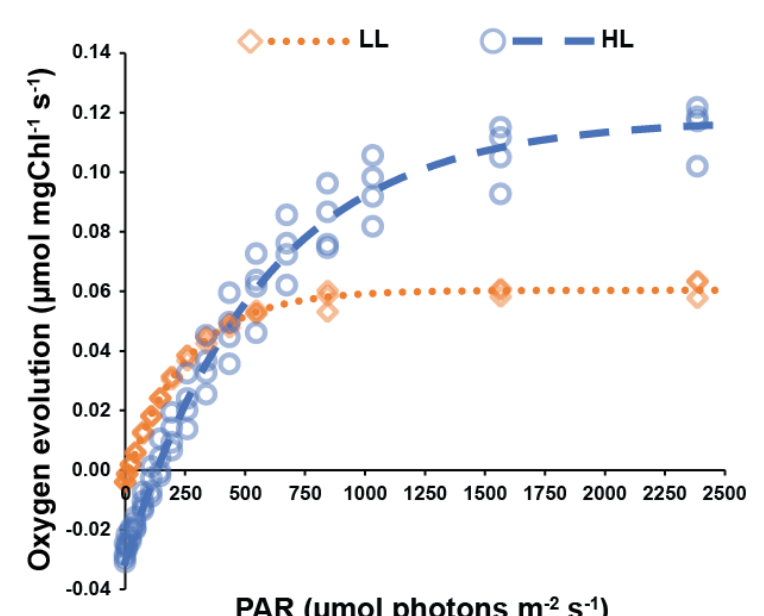

970

971

A

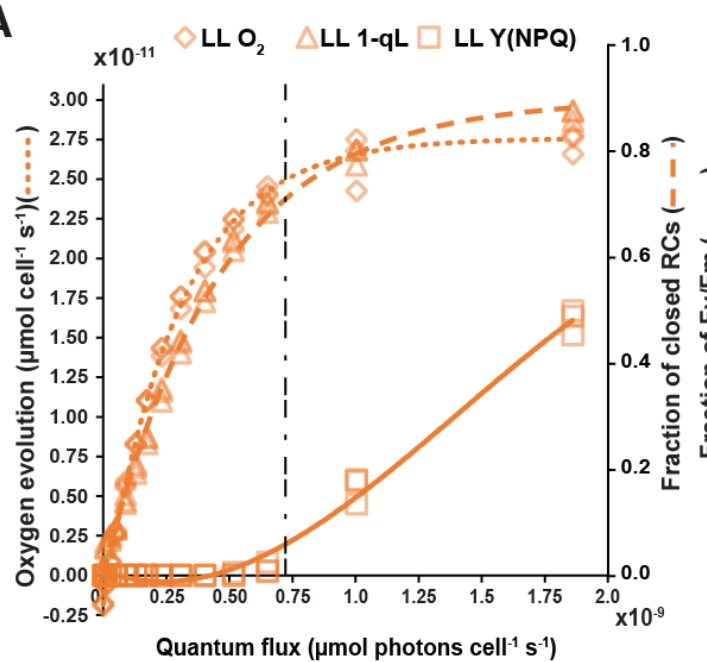

B

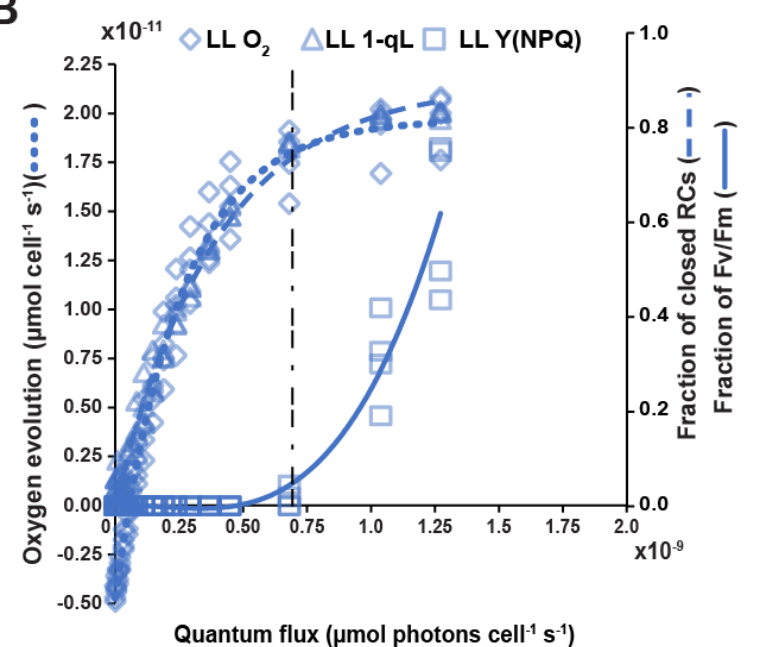

Figure S1. Chlorophyll-normalized photosynthetic rate versus quantum flux. Chlorophyll normalized $P_{O}$ versus PAR curve. Abbreviations and definitions: LL: low light, HL: high light, PAR: photosynthetically available radiation. Data based on $n=3$ biological replicates for $\mathrm{LL}$ and $\mathrm{n}=4$ biological replicates for $\mathrm{HL}$.

Figure S2. Correlation between physiology parameters at Low and High Light acclimation. (A) Oxygen evolution, 1-qL (fraction of closed reaction centers (RCs)) and $Y(N P Q)$ versus quantum flux for cells acclimated to low light. (B) Oxygen evolution, 1-qL and $Y(N P Q)$ versus quantum flux for cells acclimated to high light. Vertical dashed line indices the quantum flux value where $Y(N P Q)$ exceeds $5 \%$ of the $F_{v} / F_{m}$ fraction. 
A

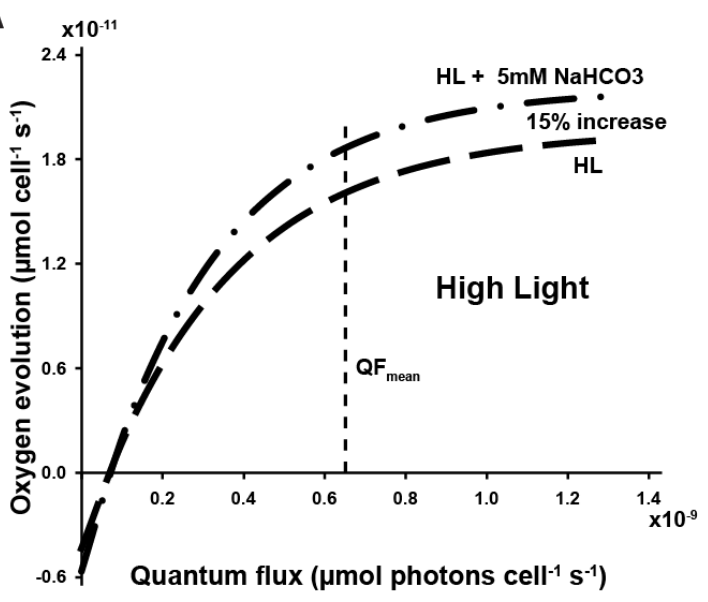

B

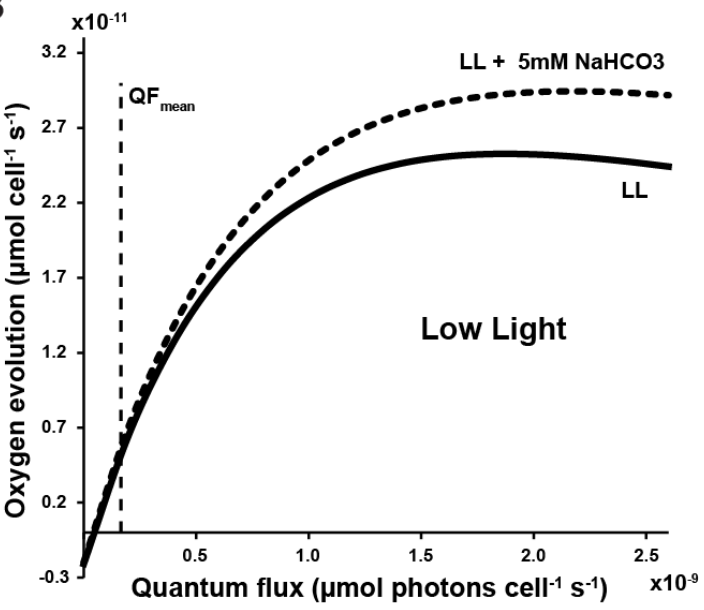

C

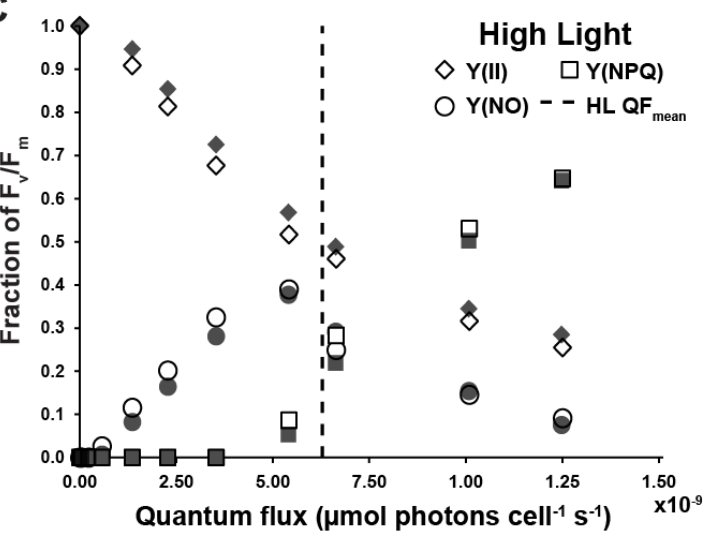

Figure S3. Impact on photosynthetic parameters with and without $5 \mathrm{mM}$ bicarbonate. (A) Cell-specific $\mathrm{P}_{\circ}$ versus $Q F$ curve for $P$. tricornutum acclimated to high light with and without $5 \mathrm{mM}$ sodium bicarbonate. Vertical dashed lines represent the quantum flux received by the cultures at the experimental irradiance. (B) Cell-specific $P_{0}$ versus QF curve for $P$. tricornutum acclimated to low light with and without $5 \mathrm{mM}$ sodium bicarbonate. Vertical dashed lines represent the quantum flux received by the cultures at the experimental irradiance. (C) Chlorophyll fluorescence measurements (PAM) of $P$. tricornutum acclimated to high light with and without bicarbonate additions. Filled in symbols: $+5 \mathrm{mM}$ sodium bicarbonate; empty symbols: $-5 \mathrm{mM}$ sodium bicarbonate. Abbreviations. HL: high light, QF: quantum flux. 


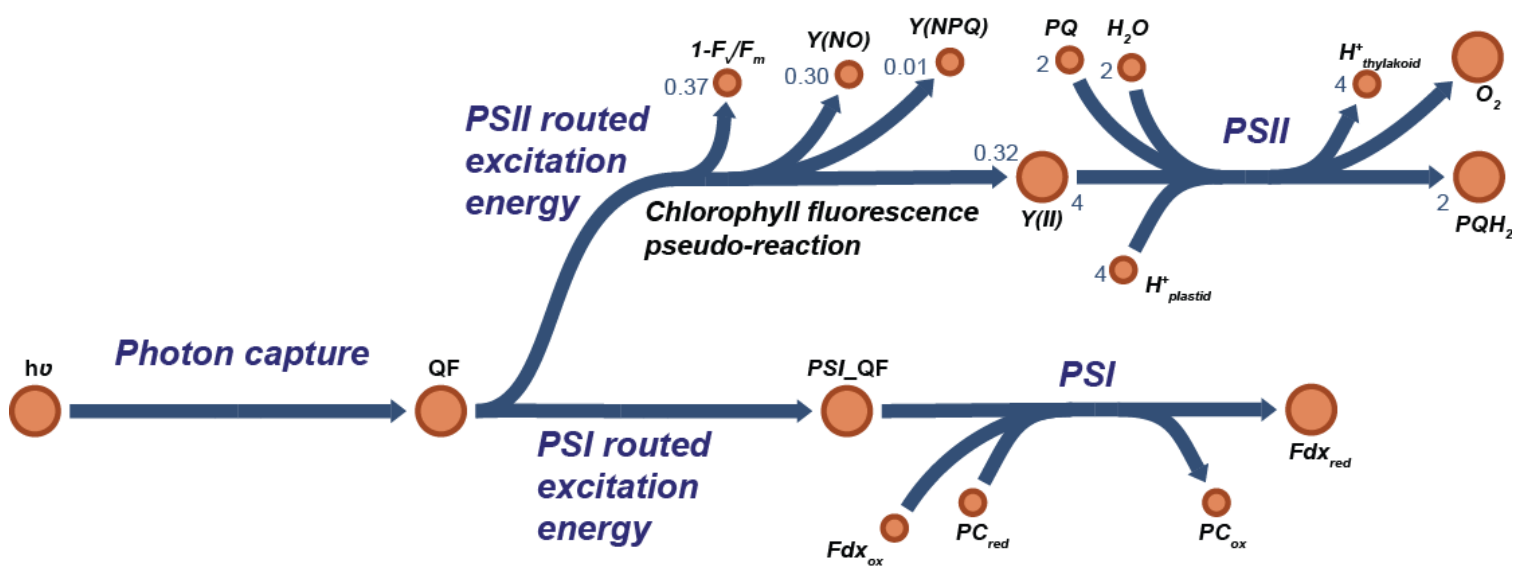

Figure S4. Incorporation of chlorophyll fluorescence parameters in the genome-scale model. Values for the pseudoreaction are representative of the high light acclimated samples. Abbreviations: hv: photon flux, QF: quantum flux, PSI_QF: quantum flux allocated to photosystem I, Fdxox/red: oxidized/reduced ferredoxin, $\mathrm{PC}$ ox/red: oxidized/reduced plastocyanin, $\mathrm{PQ}$ : oxidized plastoquinone, $\mathrm{PQH}_{2}$ : reduced plastoquinone.

A

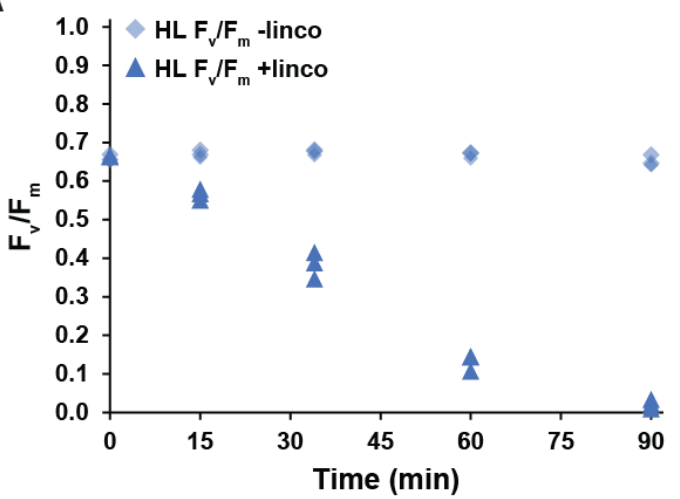

B

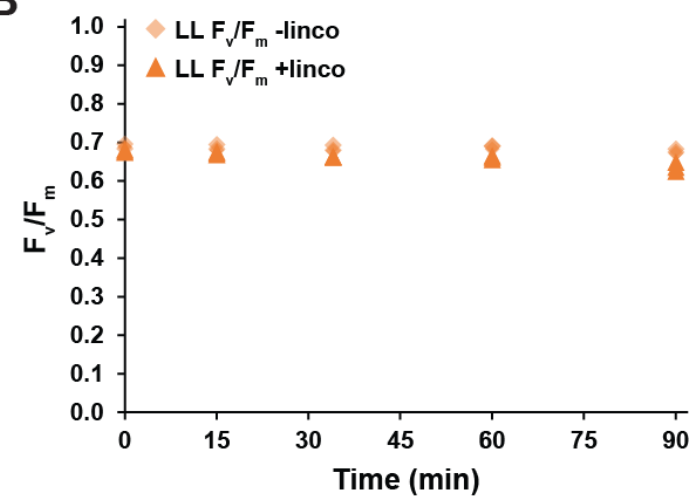

Figure S4. Maximum quantum yield of photosystem II with and without lincomycin treatment. Maximum quantum yield of photosystem II (Fv/Fm), with and without lincomycin treatment, for cells acclimated to (A) high light $(n=3)$ and $(B)$ low light $(n=3)$. 
A.

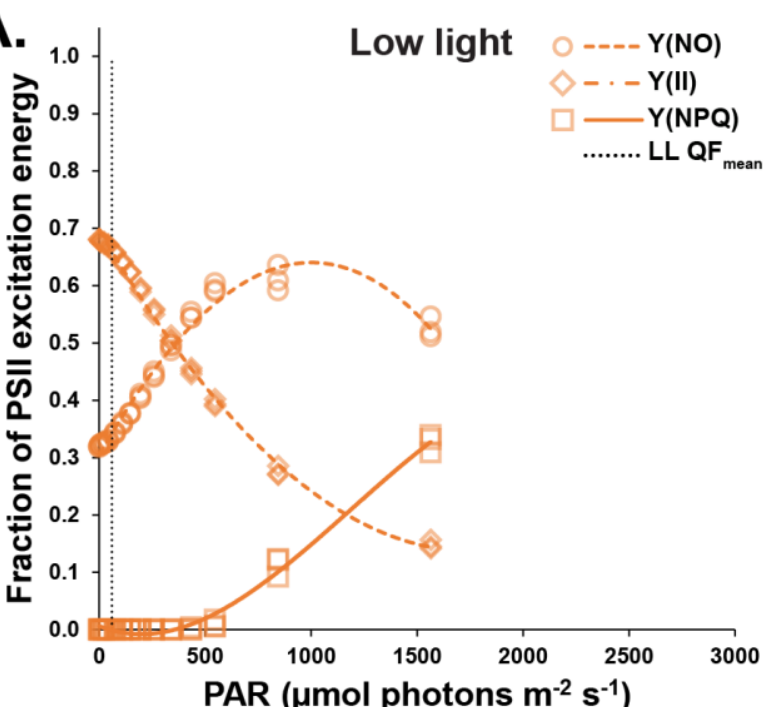

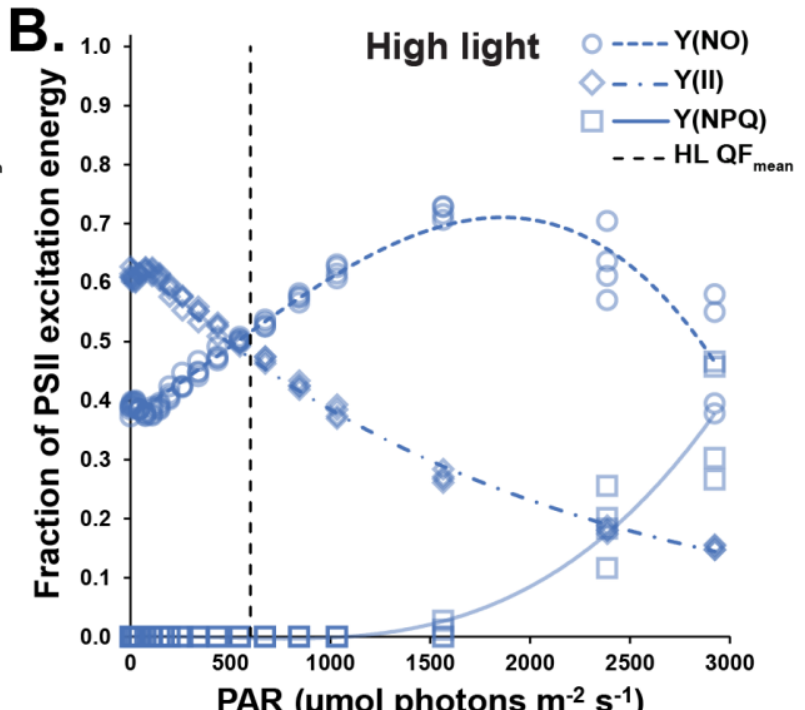

PAR ( $\mu \mathrm{mol}$ photons $\mathrm{m}^{-2} \mathrm{~s}^{-1}$ )

Figure S5. (A) Chlorophyll fluorescence parameters vs. PAR for cells acclimated to low light. (B) Chlorophyll fluorescence parameters vs. PAR for cells acclimated to high light. Vertical dashed lines represent the PAR at the experimental irradiance. Abbreviations and definitions: LL: low light, HL: high light, PAR: Photosynthetically available radiation, Y(II): quantum efficiency of photosystem II, NPQ: non-photochemical quenching, $\mathrm{Y}(\mathrm{NO})$ : unregulated, non-radiative dissipation of excitation energy. Data based on $\mathrm{n}=3$ biological replicates for $\mathrm{LL}$ and $\mathrm{n}=4$ biological replicates for $\mathrm{HL}$. 